\title{
Toric Lego: a method for modular model building
}

\section{Vijay Balasubramanian, ${ }^{a}$ Per Berglund ${ }^{b, c}$ and Iñaki García-Etxebarria ${ }^{a}$}

${ }^{a}$ Department of Physics and Astronomy, University of Pennsylvania, 209 South 33rd Street, Philadelphia, PA 19104-6396, U.S.A.

${ }^{b}$ Department of Physics, University of New Hampshire, Durham, NH 03824, U.S.A.

${ }^{c} \mathrm{PH}$-TH Division, CERN, CH-1211 Geneva 23, Switzerland

E-mail: vijay@physics.upenn.edu, per.berglund@unh.edu, inaki@sas . upenn. edu

ABSTRACT: Within the context of local type IIB models arising from branes at toric CalabiYau singularities, we present a systematic way of joining any number of desired sectors into a consistent theory. The different sectors interact via massive messengers with masses controlled by tunable parameters. We apply this method to a toy model of the minimal supersymmetric standard model (MSSM) interacting via gauge mediation with a metastable supersymmetry breaking sector and an interacting dark matter sector. We discuss how a mirror procedure can be applied in the type IIA case, allowing us to join certain intersecting brane configurations through massive mediators.

KEYwORDS: Intersecting branes models, D-branes

ARXIV EPRINT: 0910.3616 


\section{Contents}

1 Introduction and methodology 1

2 Review of dimer model technology 3

2.1 Toric geometry: web diagrams and toric diagrams 3

2.2 Gauge theories from toric singularities: quiver diagrams and dimer models 4

2.3 Zig-zag paths: From dimer models to toric geometry 5

2.4 The fast inverse algorithm: From toric geometry to dimer models 6

$\begin{array}{ll}2.5 \text { The low energy spectrum for partial resolution } & 7\end{array}$

3 Joining singularities $\quad 10$

3.1 The process of unresolving 11

$\begin{array}{lll}3.2 & \text { Tadpole cancellation } & 12\end{array}$

4 A simple dark matter model $\quad 14$

$\begin{array}{lll}4.1 & \text { Supersymmetry breaking sector } & 15\end{array}$

$\begin{array}{ll}4.2 \text { The visible/dark matter sector } & 16\end{array}$

$\begin{array}{lll}4.3 & \text { Putting the different sectors together } & 16\end{array}$

5 Some interesting modifications of the construction $\quad 18$

$\begin{array}{lll}5.1 & \text { Flavor D7 branes and an improved standard model } & 18\end{array}$

5.2 Orientifolds and improved supersymmetry breaking 20

6 Mirror description of the small resolution $\quad 24$

6.1 The mirror manifold 24

$\begin{array}{ll}\text { 6.2 The amoeba map and small resolutions } & 26\end{array}$

$\begin{array}{lll}6.3 & \text { Orientifolds and algae } & 32\end{array}$

$\begin{array}{lll}7 & \text { Conclusions and open questions } & 33\end{array}$

\section{Introduction and methodology}

Many interesting and successful classes of models for physics beyond the standard model involve various different sectors of light modes coupled only through massive mediator particles. For example, models of gauge mediated supersymmetry breaking (GMSB) are composed of a sector representing the degrees of freedom in the MSSM, and another sector breaking supersymmetry (for a review, see [1]). These sectors are coupled by massive mediators which induce soft supersymmetry breaking terms in the visible sector once we integrate out the massive fields. Recent observations by the PAMELA, ATIC and FERMI 
collaborations have also suggested the possibility of an additional interacting dark mater sector, coupled to the standard model via massive mediators [2].

It is therefore interesting to devise methods for embedding such multi-sector interacting models in string theory. From the perspective of the landscape of string theory vacua there are also statistical reasons to expect multiple interacting sectors - all we really require in a model is that below a $\mathrm{TeV}$ we should recover the standard model. Previous work has embedded two-sector models of gauge mediated supersymmetry breaking in string theory $[3-5] .{ }^{1}$ Here we extend these ideas to a systematic procedure for joining theories coming from branes at toric singularities into a consistent local embedding, such that the different subsectors interact only through massive mediators, with tunable scales. This allows us to construct string theoretic embeddings of theories with an arbitrary number of hidden sectors, such as those advocated in models of interacting dark matter [2]. From the field theoretic perspective, we are giving a systematic procedure for adding massive mediators between subsectors, such that the whole model is embeddable in string theory, with mediator masses geometrized as the sizes of resolved cycles in a singularity. Our procedure allows us to join subsectors in a modular way, and covers a large class of interesting models, such as branes at abelian orbifolds, or branes wrapping local cones over toric del Pezzo surfaces.

Our framework is a refinement of $[4,5]$ where it was shown how local singular toric Calabi-Yau geometries can be built from a slightly resolved parent singularity in order to construct multi-sector models interacting through massive matter in Type II string theory. These papers did not give a recipe for finding the particular parent theory that can be resolved to give two subsectors of choice. This was because the same geometry can support different field theories related by Seiberg duality, which is realized as toric duality in the geometry $[15,16]$. When building a particular model, one is often interested in getting a particular representative of the family of Seiberg duals after resolution - but this typically involves trial and error with many Seiberg dual phases. Instead of trying to intuit the correct parent singularity, we describe here a bottom-up approach: we give a method of splicing together sectors that realize the desired low-energy phenomenology.

The theories we construct have a very rich spectrum of massive mediators, with a plethora of both chiral and vector multiplets. Despite their complexity as low energy theories, they are remarkably simple from the string theory point of view, depending only on a small number of parameters with simple geometric realizations. Such rich mediators are generic in models arising from D-branes at sub-stringy separation.

This paper is organized as follows. Section 2 reviews some basic facts about branes at toric singularities and the corresponding dimer models that we need. Section 3 describes our algorithm for joining models and their corresponding singularities, a key technical result in this paper. Section 4 describes a particular three-sector dark matter model. Section 5 shows how the construction can be modified, including the introduction of orientifold planes.

\footnotetext{
${ }^{1}$ Alternative mechanisms for mediation of supersymmetry breaking which have been engineered in string theory include (higher form) U(1) mediation [6, 7], anomaly mediation [8-10], instanton mediation [11], holographic mediation [12] and of course gravity mediation [13, 14].
} 
a)

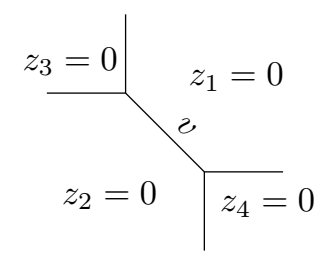

b)

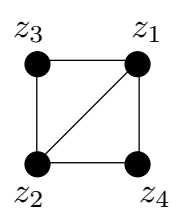

Figure 1. a) Web diagram for the conifold. We have mapped the non-compact divisors of the conifold to regions in the web diagram. $v$ denotes the resolution parameter. b) Toric diagram for the conifold.

Section 6 presents the type IIA mirror description of joining dimer models, and section 7 concludes the paper.

While this paper was being completed we became aware of [17], which contains results related to ours.

\section{Review of dimer model technology}

We start by briefly reviewing the aspects of dimer models that we use in the present work. The reader familiar with dimer model techniques can safely jump to section 3, although section 2.5 reviews less well known material. For further details, the interested reader may consult the excellent reviews by Kennaway [18] and Yamazaki [19].

\subsection{Toric geometry: web diagrams and toric diagrams}

Let us start by recalling some elementary facts about toric Calabi-Yau threefolds. We use the conifold as our example, with other toric varieties constructed in a similar fashion. In its singular limit, the conifold is defined by the equation

$$
\sum_{i=1}^{4} z_{i}^{2}=0
$$

inside $\mathbb{C}^{4}$. This geometry is toric, meaning that it admits a $\mathrm{U}(1)^{3}=T^{3}$ fibration, this can be seen as follows. Equation (2.1) is invariant under $\mathrm{SO}(4)$ rotations transforming the $z_{i}$ as a vector, and also invariant under $\mathrm{U}(1)$ rotations of the four coordinates, sending $z_{i} \rightarrow e^{i \theta} z_{i}$. This gives a symmetry group $\mathrm{SO}(4) \times \mathrm{U}(1) \sim \mathrm{SU}(2) \times \mathrm{SU}(2) \times \mathrm{U}(1) \supset \mathrm{U}(1)^{3}$, so the conifold is indeed a toric variety. In our examples, we are interested in a smooth space connected to the singular conifold described above, the resolved conifold, obtained by blowing up a two sphere of size $v$ at the singularity of the conifold.

It turns out that all the relevant information about the geometry can be efficiently encoded by specifying over which points in the (three real dimensional) base the $T^{3}$ fiber degenerates. For the case of Calabi-Yau manifolds, this information can be easily visualized in terms of a web diagram. The web diagram is a projection of the base of the toric manifold onto a plane. The base is a three real dimensional polyhedron with a $T^{3}$ fiber over each point - the fibers degerate over faces and edges of the polyhedron which appear in the planar projection of the polyhedron (see [20] for a review). For our purposes it suffices 

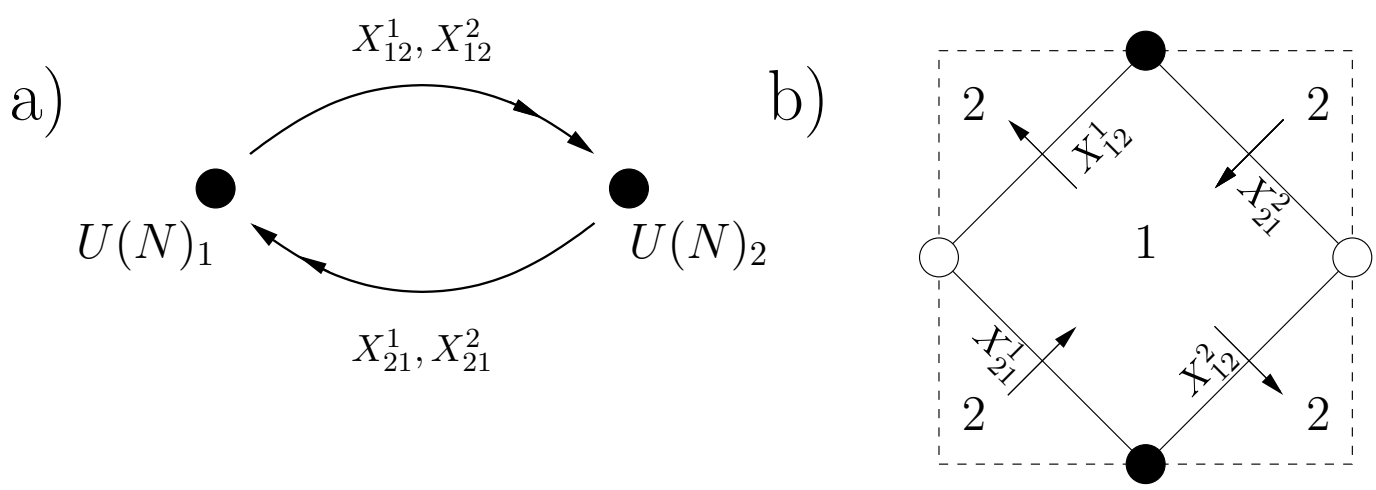

Figure 2. a) Quiver diagram for the conifold. The arrow with two heads represents two bifundamentals. b) Dimer model for the conifold. Note that the opposite sides of the square should be identified, forming a two-torus, i.e., there is a single black dot and a single white dot. We have denoted the orientation of the edges by arrows perpendicular to them: an arrow from $\mathrm{U}(N)_{a}$ to $\mathrm{U}(N)_{b}$ crossing the edge $E$ means that $E$ transforms in $\left(\sqsubset l_{a}, \bar{\square} l_{b}\right)$. We have also labelled the bifundamentals as in the text.

to know that every manifold posses a web diagram, which is a graph drawn on a integer lattice. The web diagram for the resolved conifold is given in figure 1. The singular conifold is recovered by shrinking away the edge marked $v$. We also show its planar dual, the toric diagram, which encodes the same information and is often more useful to work with.

\subsection{Gauge theories from toric singularities: quiver diagrams and dimer models}

Let us now proceed to put a stack of $N$ D3 branes on the singular point $z_{i}=0$. As argued by Klebanov and Witten [21], the resulting theory is $\mathrm{U}(N)_{1} \times \mathrm{U}(N)_{2}$ with four bifundamentals ${ }^{2} X_{12}^{i}, X_{21}^{i}(i$ takes values in 1,2$)$, and a superpotential:

$$
W=\operatorname{Tr}\left(X_{12}^{1} X_{21}^{1} X_{12}^{2} X_{21}^{2}-X_{21}^{2} X_{12}^{2} X_{21}^{1} X_{12}^{1}\right)
$$

It is helpful to represent this information graphically. The most common representation is in terms of quiver diagrams. These are oriented graphs in the plane where nodes represent gauge groups, and bifundamentals fields are arrows connecting the nodes. We show the quiver diagram for the conifold in figure 2a. This representation of the theory, however, misses the F-term information encoded in the superpotential. It turns out that for theories coming from D3 branes located at toric singularities, such as the conifold, it is possible to do much better, using dimer models [22]. A dimer model is a periodic tiling of $\mathbf{R}^{2}$ (equivalently, a tiling of $T^{2}$ ) with the property that nodes in the graph can be colored black or white, and white nodes only connect to black nodes (and vice versa). The dictionary between this tiling and properties of the associated gauge theory is as follows:

\footnotetext{
${ }^{2}$ We always use the notation $X_{a b}^{\bullet}, Y_{a b}^{\bullet}, \ldots$ to denote fields transforming in the fundamental representation of $\mathrm{U}(N)_{a}$ and the antifundamental of $\mathrm{U}(N)_{b}$.
} 
- Faces represent $\mathrm{U}(N)$ gauge groups. ${ }^{3}$ In the conformal case we have discussed so far all faces have the same rank, but we will see below that it is also possible that faces have different rank, representing D5 and D7 brane charge localized at the singularity (i.e., fractional D3 charge).

- Edges between faces $\mathrm{U}(N)_{a}$ and $\mathrm{U}(N)_{b}$ represent bifundamental fields. It is possible to assign a unique orientation to the edges (whether they transform in $\left(\left.\sqsubset\right|_{a}, \bar{\complement}_{b}\right)$ or in $\left.\left(\bar{\complement}_{a}, \sqsubset b_{b}\right)\right)$ by imposing that edges around a black node go clockwise and around a white node counter-clockwise. Note that this implies that as we go around a face, edges alternate in their orientation. This also implies that every face has an even number of edges, half incoming and half outgoing.

- Finally, nodes represent superpotential terms (more precisely, monomials in the superpotential). The rule to obtain the precise monomial is to multiply the fields around a black node clockwise, with an overall plus sign, and multiply fields around a white node counter-clockwise, with an overall minus sign. One should take the trace of the resulting polynomial in order to obtain a gauge invariant superpotential.

We have shown the quiver diagram and dimer model for the conifold in figure 2 . It is a simple exercise to verify that with the rules given here the dimer model in figure $2 \mathrm{~b}$ encodes precisely the conifold theory described above. In particular, the superpotential is given by (2.2).

At this point two natural questions arise: can we obtain the dimer diagram for a given toric singularity in a simple way? Is the geometry of the toric variety naturally encoded in the dimer diagram? ${ }^{4}$ Surprisingly, the answer to both questions is affirmative, the essential concept being the zig-zag path [25, 26]. We now review the definition and properties of zig-zag paths, which are an essential tool in our construction, and show how they naturally give an elegant answer to the second question. We postpone the answer to the first question to section 2.4.

\subsection{Zig-zag paths: From dimer models to toric geometry}

Zig-zag paths [25, 26] (also called rhombus paths in the context of isoradial embeddings) are paths in the dimer model with the property that they turn maximally at each node, and they cross once the edges along which they run. We illustrate a typical zig-zag path in figure 3a (see also figure 4 for the set of zig-zag paths for the conifold).

Zig-zag paths have a number of interesting and useful properties, of which we mention a few here. We do not provide justification for the following statements, but refer the reader to the original papers [25-27].

\footnotetext{
${ }^{3}$ In string theory most of the U(1) factors actually decouple at low energies, getting mass by mixing with background $R R$ axions [23, 24], although some of the anomaly-free $\mathrm{U}(1)$ factors can remain massless. For simplicity, we always keep these U(1) factors around, and just discuss their fate when it makes a difference.

${ }^{4}$ The toric variety should arise as the moduli space for the theory of a single regular brane located at the singular point, so the dimer model does encode all the information about the toric singularity. The question is how to determine this moduli space efficiently from the dimer model.
} 
a)

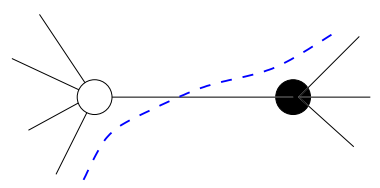

b)

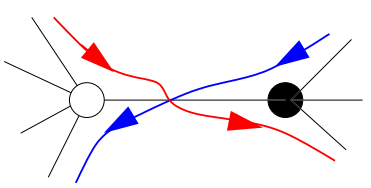

Figure 3. a) Generic zig-zag path, drawn as a dashed blue line. It turns maximally when it reaches an edge, and it crosses the edges along which it runs. b) When two zig-zag paths intersect, they do it over an edge and running with opposite orientations.

The first property is that each edge in the dimer model corresponds to an intersection of two zig-zag paths. That is, every time two zig-zag paths intersect they give rise to a bifundamental field.

A second property is that we can naturally assign an orientation to each zig-zag path. In order to do this, assign arbitrarily an orientation to one of the zig-zag paths. The orientation of the rest of the zig-zag paths is determined by the requirement that when two zig-zag paths intersect over an edge, they do it with opposite orientations, see figure $3 \mathrm{~b}$.

We can now describe how zig-zag paths solve the second problem above, namely how to obtain the toric variety from the dimer model. Take one zig-zag path, with its orientation. Compute its $(p, q)$ winding number along the torus on which the dimer model is defined. This can be represented as a vector in the plane, pointing in the $(p, q)$ direction. Once we do this for all zig-zag paths, we obtain the web diagram of the (unresolved) toric singularity. ${ }^{5}$ As an example, in figure 4 we compute the web diagram of the conifold from the conifold dimer model.

\subsection{The fast inverse algorithm: From toric geometry to dimer models}

Let us now proceed to describe how one can compute the dimer model corresponding to a given toric singularity. The most efficient algorithm, and the one that will inspire our joining algorithm in section 3 , is the fast inverse algorithm, first described in [26]. Since we will make use of some of the ideas of this algorithm in the following sections, let us briefly describe how it works.

The idea is simple and uses the key concept of zig-zag paths: draw in a torus cycles with homology charges corresponding to the external legs of the geometry we want to consider. These cycles divide the torus into regions, with boundaries given by portions of zig-zag paths. These regions can be divided into two classes: those around which the boundary has a definite orientation (inherited from the zig-zag paths defining the boundary), and those which have no definite orientation. From this information we can easily recover the dimer model, simply by identifying oriented regions with superpotential terms, zig-zag path intersections with edges, and unoriented regions with gauge factors. We show how this works in the case of the conifold in figure 5. Let us note that we could have further subdivided oriented regions into clockwise and counter-clockwise regions. As it is simple to see from examples, this corresponds to the black/white coloring of the nodes in the dimer model.

\footnotetext{
${ }^{5}$ This can be naturally understood using mirror symmetry [27]. See also section 6 .
} 
a)

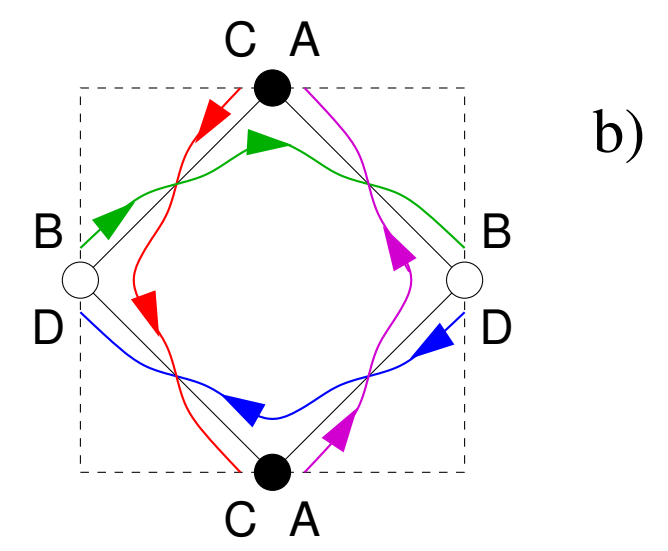

b)

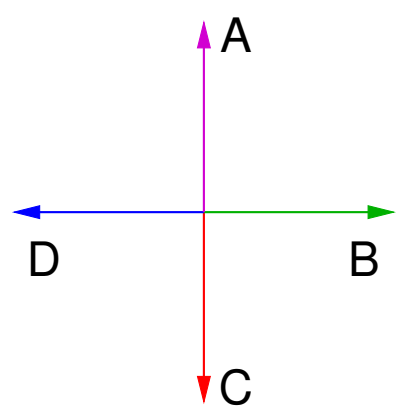

Figure 4. a) Set of zig-zag paths for the conifold. b) The web diagram obtained out of the zig-zag paths.

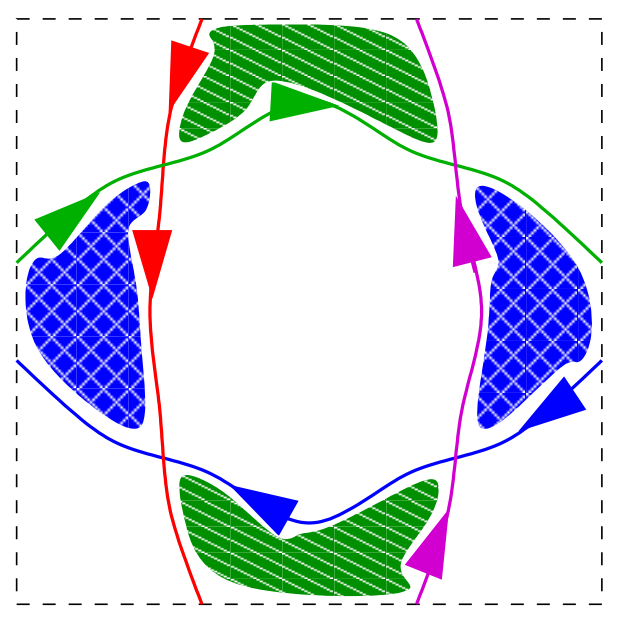

Figure 5. Inverse algorithm for the conifold. The two shaded regions are oriented, and correspond to the two superpotential terms, while the two regions not shaded do not have a definite orientation, and correspond to the two faces of the dimer model. Intersections of zig-zag paths correspond to bifundamental fields.

\subsection{The low energy spectrum for partial resolution}

Zig-zag paths have another very interesting application. As mentioned above in section 2.1, it is possible to smooth out the singularity in the conifold by blowing up a two-cycle. Similarly, any toric Calabi-Yau variety can be completely smoothed out by blowing up two and four cycles at the singularity. A complete resolution results in a toric diagram which is maximally triangulated, and alternate triangulations describe resolutions related by flops. It is always possible to completely smooth out a toric Calabi-Yau in this way, but it is not necessary to completely smooth it out, partial resolutions are also possible. In such a case, one blows up one of the zero-size two-cycles, while leaving parts of the geometry singular. Each such partial resolution partitions the toric diagram into concatenated convex polytopes.

As a simple two complex dimensional example that illustrates the idea of partial res- 
olution, consider the geometry defined by the equation:

$$
f=x^{2}+y^{2}+z^{4}=0
$$

in $\mathbb{C}^{3}$. This geometry has a singularity (of type $A_{3}$ ) at the origin, due to the fact the $f=d f=0$ there. It is possible to blow up a two-cycle and make the geometry less singular by modifying the defining equation to:

$$
\widetilde{f}=x^{2}+y^{2}+z^{2}(z-a)^{2}=0
$$

with $a \neq 0$. The new geometry has two singularities of lower rank (they are now of type $\left.A_{1}\right)$ at $x=y=z=0$ and at $x=y=0, z=a$. The toric threefold examples we construct behave in a similar way. We start with a "big" singularity, and partially blow it up in order to obtain a Calabi-Yau variety with interesting "smaller" singularities at a finite distance from each other.

In our constructions the gauge theory comes from branes located at the singularity, so we need to know how the geometric operation of resolving the singularity affects the gauge theory living on the branes. This problem was solved for arbitrary resolutions of toric Calabi-Yau varieties in $[4,5]$. As in the rest of this review section, we merely present the rules for obtaining the answers, leaving the justification to the original references.

Let us review the algorithm for obtaining two dimer models corresponding to the massless matter living on the branes at the two remaining daughter singularities, and the massive mediators coupling the two massless sectors. In order to fix notation, let us call the two daughter singularities 1 and 2. As noted above, we can naturally split the external legs of the toric diagram into two sets, associated to the two daughter singularities. This naturally gives us a splitting of the zig-zag paths for the mother singularity into two sets. Let us abuse notation and also call these sets 1 and 2 (which particular meaning of " 1 " and "2" we are talking about will be clear from the context).

Having split the zig-zag paths into two sets, associated to the two daughter singularities, we can classify the set of edges in the parent dimer into three sets: those over which two zig-zag paths of type 1 intersect (edges of type 1), those over which zig-zag paths of type 2 intersect (edges of type 2), and edges over which a type 1 zig-zag path and a type 2 zig-zag path intersect (edges of type 3 ).

The result in $[4,5]$ states that the correct field theory description of the resolution of the singularity is to give the following sets of vevs to the different types of bifundamentals:

$$
\left\langle X^{1}\right\rangle=\left(\begin{array}{cc}
0 & 0 \\
0 & v \mathbb{I}_{Q}
\end{array}\right), \quad\left\langle X^{2}\right\rangle=\left(\begin{array}{cc}
v \mathbb{I}_{P} & 0 \\
0 & 0
\end{array}\right), \quad\left\langle X^{3}\right\rangle=0
$$

where we have denoted edges of type $i$ as $X^{i}$, and we have split the $N$ branes at the original singularity into $P$ branes going to the daughter singularity 1 , and $Q$ going to the daughter singularity $2 . \mathbb{I}_{P}, \mathbb{I}_{Q}$ denote the identity matrices of rank $P, Q . v$ parameterizes the size of the blown-up cycle. For simplicity, we have presented the case in which all the faces of the original dimer have the same rank $N$ (corresponding to the case with vanishing fractional brane charge), so the vevs for the $X^{i}$ bifundamentals can be naturally represented as 
a)

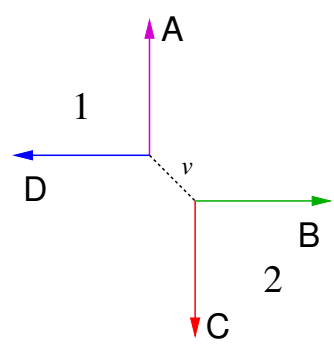

b)

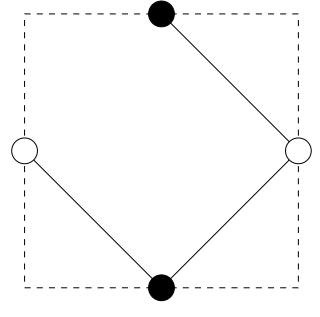

c)

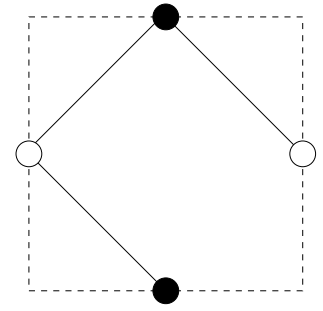

Figure 6. Resolution process for the conifold into two copies of flat space. a) Resolution in terms of the web diagram. The internal dashed lines denotes the blown up cycle, of size $v$. We have denoted the two daughter singularities 1 and 2, as in the text. b) Dimer model corresponding to the daughter singularity 1 , obtained by removing the edge where B and C intersect (see figure 4a). c) Dimer model for the daughter singularity 2 , obtained by removing the edge where A and D intersect. Note that both b) and c) match what one would obtain by directly applying the fast inverse algorithm to flat space.

$N \times N$ matrices. The case with non-vanishing fractional charge works similarly (see $[4,5]$ for details).

This prescription admits a very simple description in terms of dimer models: edges of type 1 disappear from the dimer model for the daughter singularity 2 (representing the Higgsing coming from the nonzero vev), edges of type 2 disappear from the dimer model for the daughter singularity 1 , and edges of type 3 remain in both daughter dimers. We illustrate this procedure for the case of the conifold in figure $6 .^{6}$

The procedure we are describing can always be carried out for gauge theories arising from D3-branes at a toric singularity. In this case every gauge group in the parent theory has rank $N$ while the gauge factors at the two daughter singularities all have ranks $P$ and $Q$ with $P+Q=N$. If fractional branes are wrapped on any of the collapsed cycles of the parent singularity, the resolutions of these cycles do not lie on the moduli space and cannot be carried out. In other words, in the presence of fractional branes a partial resolution can only be carried out if these branes are wrapped on cycles that are entirely carried into one of the daughter singularities and not on the cycle we are blowing up. Fortunately, in this paper we will always go in the other direction - given daughter singularities with or without fractional branes we will give a gluing prescription that will give a larger parent singularity containing both of the daughters. Thus, for our purpose of describing the spectrum of the complete theory we can assume that the blowup in question can be performed even in the presence of fractional branes.

Given the gauge theory of the parent singularity, and explicit expressions for the vevs of the bifundamentals responsible for the partial resolution, we can give a complete description of the theory after the resolution, including the massive mediator sector, with its couplings. There are some simple rules for determining the massive spectrum, which apply even in

\footnotetext{
${ }^{6}$ In the case of the conifold a single blow-up completely smooths out the space, giving two copies of flat space, so the term "daughter singularity" is perhaps a bit misleading in this context. The procedure still applies without any change, and gives the right answer: $\mathcal{N}=4$ super-Yang-Mills in $\mathcal{N}=1$ notation, as it is easy to check from figures $6 \mathrm{~b}$ and $6 \mathrm{c}$.
} 
the presence of fractional branes as long as the resolution is possible:

1. Consider an edge which disappears in the $i^{\text {th }}$ daughter dimer diagram leaving a face with a gauge group of rank $K$. Then there is also a massive vector multiplet in the adjoint of $\mathrm{U}(K)$ gauge factor. This massive multiplet arises from Higgsing of a $\mathrm{U}(K) \times \mathrm{U}(K)$ subgroup of the gauge factors of the two faces the edge used to separate in the parent theory. Only the diagonal component of this subgroup survives the Higgsing, while the non-diagonal part gets a mass through the Higgs mechanism.

2. For each face in the parent dimer diagram, we obtain two massive vector multiplets in the bi-fundamental $\left(N_{1}, \overline{N_{2}}\right)$ and its conjugate, where $N_{1,2}$ are the ranks of the faces in the daughter dimers that enclose the face in the parent. (It might be that the faces in the daughters have expanded via the loss of edges, but they never shrink in the procedure we are describing.)

3. Consider an edge present in both daughter dimer diagrams separating faces with ranks $N_{1}^{a}, N_{1}^{b}$ and $N_{2}^{a}, N_{2}^{b}$ in the two daughters respectively. Also suppose that the edge was oriented in the parent so that bifundamentals went from $a$ to $b$. Then in the daughter theory there are massive chiral multiplets in $\left(N_{1}^{a}, \overline{N_{2}^{b}}\right)$ and $\left(\overline{N_{1}^{b}}, N_{2}^{a}\right)$ bifundamental representations. The dimer diagram ensures that globally, these types of chiral multiplets pair up consistently to form massive scalar multiplets [5].

4. If the daughter dimer diagrams contain bi-valent nodes (nodes with two edges), the pair of edges gives a massive scalar multiplet in the bi-fundamental of the two faces separate by the pair.

If there are non-compact D7-branes threading the singularity, additional massive matter will appear (see section 5 for details.)

\section{$3 \quad$ Joining singularities}

In the preceding section we discussed the process of partially resolving a larger (mother) singularity into several smaller (daughter) singularities. We now consider the reverse process, i.e., unresolving or joining daughter singularities to form a new singularity. There are several reasons why this may be a more useful way to proceed. First, from a bottom-up approach we start with a low-energy theory that consists of several sectors, each of which is described by putting D-branes on a singularity. We then want to know how these singularities fit together, such that we can determine the massive spectrum of messenger particles. In addition, the process of splitting singularities described in section 2.5 has an ambiguity in the case that the web diagram of the parent geometry has parallel external legs of the same orientation that get split. In such a case one is free to choose which zig-zag path goes to which side of the split, and the resolution procedure we described in section 2.5 will give different answers depending on our choice. This phenomenon is a manifestation of Seiberg duality [15], which in this context appears as toric duality [16]. More generally, there exist different Seiberg-dual dimer models describing the same geometry. (Note that the conifold 
a)

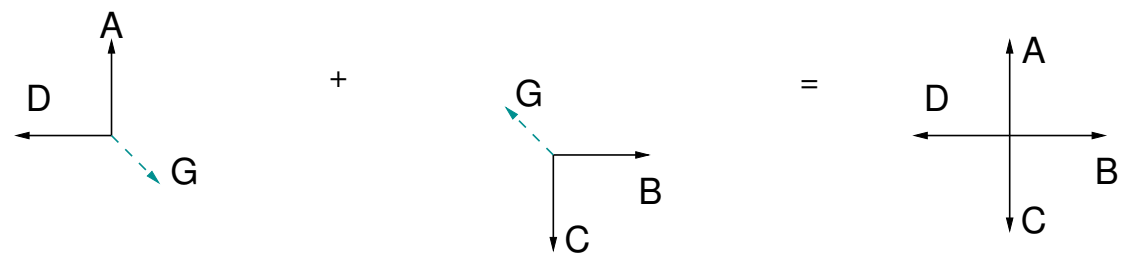

b)
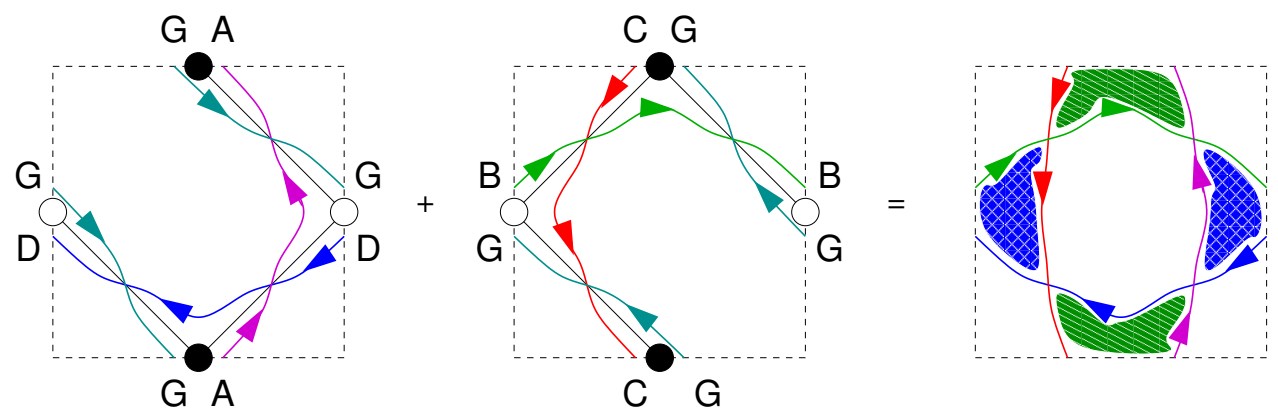

Figure 7. Joining two copies of flat space along one external leg. a) Web diagram perspective. We have indicated the external edge to be joined $(G)$ by the dashed colored line. b) Dimer model perspective.

is special since taking the Seiberg dual of any face of the dimer brings us back to the same dimer, i.e., it has a single Seiberg phase).

This effect is important when building particular models, since upon resolving the daughter singularity in different ways (thus getting Seiberg dual daughters), or upon starting with a different Seiberg dual phase of the mother theory, one obtains different daughter theories. The latter are usually different from the theories one is interested in putting together. In this section we describe a procedure, a simple combination of the fast inverse algorithm described in section 2.4 and the partial resolution algorithm described in section 2.5, that allows us to join any two singularities in such a way that upon resolution we obtain the two desired dimer models for the two daughter singularities.

\subsection{The process of unresolving}

Let us start with the dimer models that we want to join. For illustration, we reconstruct the conifold theory out of two copies of flat space. The complete procedure is shown in figure 7. This particular example does not exhibit any of the subtleties having to do with Seiberg duality, but the examples in section 4 do, and they can be analyzed in the same way. In the general case, in addition to specifying the toric singularities that we want to join, we also should choose the particular dimer model representative of the daughter singularity that we want to join.

Once we have picked the singularities, we need to choose along which external leg of their web diagram we want to join them. In general, we might need to perform a $\operatorname{SL}(2, \mathbb{Z})$ transform of the daughter dimer models in order to align the legs that we want to join. This is always possible if the legs are primitive vectors (i.e., for each vector the components are relatively prime). If a leg is non-primitive, we write it as a multiple of a primitive vector 
and then the web diagram can only be joined to another diagram with a leg that is the same multiple of an oppositely oriented primitive vector. In this case, in addition to the two sectors we want to join, we might have to attach additional daughter singularities in order to ensure that the combined parent singularity remains toric (we will see examples of this later). The new sector will not contribute massless degrees of freedom if we do not put any brane at the corresponding daughter singularity, but it will modify the precise form of the massive mediators and their couplings to massless modes.

The next step in our graphical algorithm for joining theories is to remove from each daughter dimer model everything except the external zig-zag paths that survive in the parent. ${ }^{7}$ In particular, this removes the zig-zag paths corresponding to the daughter legs that we are going to join. In the case of the conifold in figure 7 , this means leaving only the A,D zig-zag paths in the first dimer model, and B,C in the second dimer model, removing all nodes, edges, and and the zig-zag path G.

Finally, we superpose the two sets of remaining zig-zag paths, and compute the new theory as in the inverse algorithm. By construction, the new theory descends to the two dimer models we started with upon partial resolution. ${ }^{8}$

In this graphical algorithm we started by removing all the edges and nodes in the daughter dimer, and then reconstructed the dimer from the zig-zag paths of the parent theory produced by the joining algorithm. Of course we could have simply left the daughter edges and nodes in, since the joining algorithm essentially introduces a few new bifundamentals (edges) and superpotential terms (nodes). It is simply easier as a graphical technique to proceed as we described, and to reconstruct the dimer model for the joined theory from scratch.

\subsection{Tadpole cancellation}

The previous algorithm works without any further subtleties in the case that all faces in the final (joined) dimer model have the same rank. Note that this condition is equivalent to each subsector having all its faces of the same rank (although different subsectors can have different ranks). When all faces have the same rank our dimer model defines a superconformal field theory [22]. For model building applications we require theories that are non-conformal. We can achieve this in two ways: (1) by putting fractional branes in the singularity, and/or (2) by introducing non-compact D7 branes in the geometry. Let us analyze each of these possibilities in turn.

The first possibility consists of choosing the ranks of the faces in the dimer model to be different. This situation is usually referred to as putting fractional branes in the

\footnotetext{
${ }^{7}$ The resulting diagram is closely related to what is referred to as a harlequin diagram in [28], the only distinction being that we remove from the harlequin diagram the zig-zag that is going to join the singularities.

${ }^{8}$ There is still some ambiguity in how to superpose the two sets of zig-zag paths. Namely, we could deform the zig-zag paths in such a way that we induce no Yang-Baxter transform (i.e. Seiberg duality coming from continuation across triple intersections of zig-zag paths [26]) in any of the daughter dimer models, but we induce one such Yang-Baxter transform in the mother theory. This is perfectly consistent, and reflects the fact that even if the daughter singularities have their Seiberg dual phase fixed, this does not fix uniquely the Seiberg dual phase of the mother theory.
} 
system, since this kind of rank assignment comes from wrapping D5 and D7 branes on the vanishing cycles (the configuration with all ranks equal corresponds to putting a stack of D3 branes at the singularity).

In such a configuration local tadpole cancellation becomes an important issue. The condition for tadpole cancellation in $\mathcal{N}=1$ quiver gauge theories is that every node in the quiver has the same number of fundamental and antifundamental chiral multiplets. ${ }^{9}$ It is easy to see that this holds in dimer models with faces of equal rank: recall that in a dimer model, due to the alternating black/white coloring of the nodes, each face has to have an even number of edges, with alternating orientations, so each face has as many fields in the fundamental as in the antifundamental.

Once the ranks of the gauge groups are not equal, tadpole cancellation is no longer guaranteed, and we need to check it for each rank assignment. However, as long as the theories that we join are tadpole free, the joined singularity appears to be tadpole free too. Heuristically, the reason why it is enough to satisfy tadpoles in each subsector is that the compact cohomology charge adds simply when we join singularities. However, we do not have a complete proof of this observation, and it is certainly no longer true if we add non-compact D7 branes into the problem, which is often necessary to construct realistic models. In this case, anomaly cancellation in the joined theory must be checked by hand.

The reason for this is that the requisite D7 branes naturally extend along the external legs of the web diagram. When we join singularities we might be forced, to have consistency of the construction, to introduce D7 branes along subsectors that did not have them originally. As an example, let us consider sectors $A$ and $B$ where $A$ had some D7 branes, while $B$ did not. Upon joining $A$ with $B$, the D7 branes of $A$ might extend along the leg that connects $A$ to $B$ and thus have to exit the web diagram along an external leg of $B$. This introduces new flavors charged under the fractional branes in $B$. In general this also introduces local tadpoles in the theory $B$. More geometrically, the D7 brane has local tadpole charge under the compact homology of the new subsector, and we need to cancel this by adding extra fractional D-branes in the $B$ sector.

Thus, we are forced to change sector $B$ by changing the ranks of the gauge groups, and by adding extra flavors to it. In some cases this modified model might be just as good phenomenologically as the original. In general the D7 tadpole condition introduces an important constraint that the subsectors must satisfy. Even if the constraint is important, it is certainly possible to build interesting models that satisfy it (see [5] for some examples).

Finally, having obtained the tadpole free dimer model for the parent theory resulting from joining daughter singularities, we are left to determine the ranks of the gauge groups in the parent related to the ranks in the daughters. Every face in the parent dimer diagram descends to part of face in each daughter diagram (the faces in the daughter arise from removing some edges in the parent). The rank associated to the parent face is the sum of the ranks of daughter faces that it participates in.

\footnotetext{
${ }^{9}$ This condition is the same one as anomaly cancellation of the resulting gauge theory, with the addition that empty nodes must be anomaly free too. A simple way of understanding this extra condition is that we can "fill in" the empty nodes without changing the compact tadpoles by bringing a D3 brane into the singularity. This adds one unit to the rank of all faces, reducing the check for consistency to usual anomaly cancellation.
} 


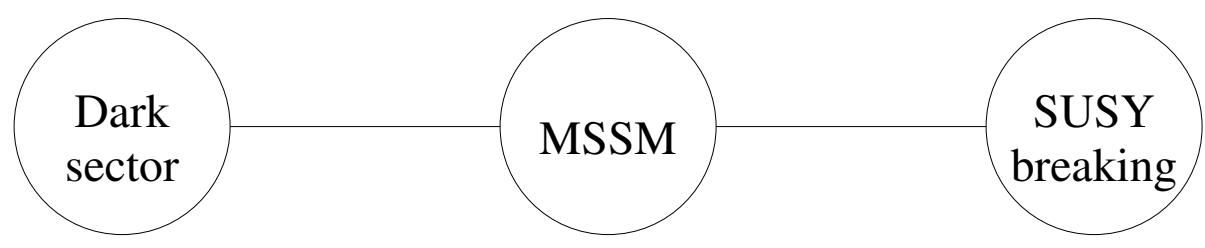

Figure 8. Schematic representation of our simple model. The nodes represent the different subsectors of light degrees of freedom. The lines connecting the different nodes represent massive multiplets charged under the different nodes.

\section{A simple dark matter model}

With these tools at hand, we can construct local models of dark matter. Our constructions are motivated by simplicity, to illustrate our methods, and phenomenologically better choices certainly exist. Because our constructions are modular, such improvements are easily incorporated, as long as they are toric. We present a number of different alternatives to this model in section 5 .

Our model consists of three sectors, coupled by massive mediators: a supersymmetry breaking sector, a "MSSM" visible sector, and a dark matter sector, which we take to be a copy of the MSSM sector, with the only difference that it couples differently to the supersymmetry breaking sector. We show the structure of the resulting theory in figure 8 . We achieve this using the techniques described in previous sections: we locally engineer each of the sectors as a dimer model, and use toric techniques to paste them together into a slightly resolved bigger singularity. In fact, a big part of the work has already been done for us in [5], where a local configuration with two sectors implementing gauge mediated supersymmetry breaking was described. We explain how to attach a third sector containing dark matter to the theory found in [5].

In more detail, our visible sector is of the trinified form ( $\mathrm{SU}(3)^{3}$ gauge group), and the dark matter sector is an exact copy of the standard model, as in the well studied mirror world proposal of [29] (adapted for trinification). We take the supersymmetry breaking sector to be a theory with a runaway potential and a metastable vacuum at the origin of moduli space. In section 5.2 we will replace this supersymmetry breaking sector by a better behaved geometry.

The resulting model is of the "minimal superdark moose" form described in [30]. In such a model, in order to get weak scale dark matter we want the same mechanism to set the $\mu$-term in the MSSM and the dark sector. Thus, the $\mu$-term has to be generated by a common mechanism (such as a D-brane instanton [31-34]) that can appear in the local geometries of both the dark sector and MSSM, rather than by RG running of the interactions with the SUSY breaking sector. Our toy models will not generate the $\mu$ term non-perturbatively, but in other local models studied in the literature this can be arranged. See for instance [34] for a explicit set of semi-realistic models from branes at toric singularities in which D-brane instantons generate the $\mu$ term non-perturbatively. 

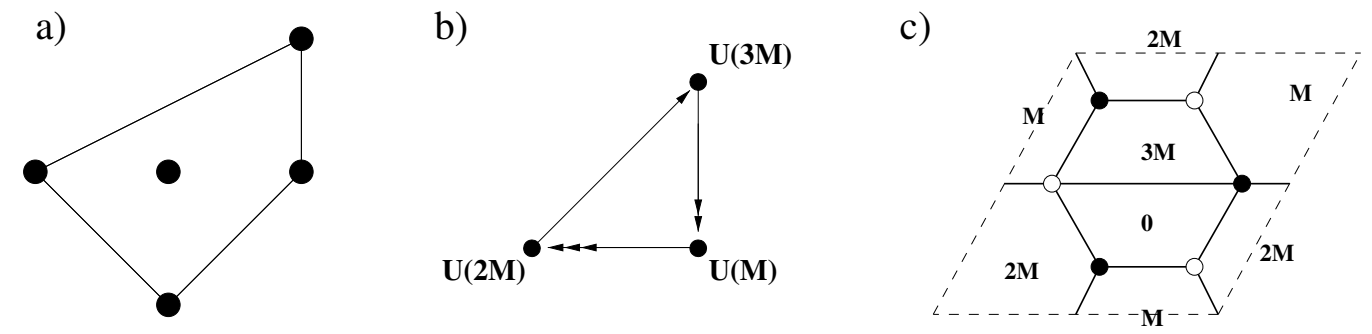

Figure 9. a) Toric diagram for the complex cone over $d P_{1}$. b) The quiver gauge theory for $M$ DSB branes in the complex cone over $d P_{1}$. c) Dimer model.

\subsection{Supersymmetry breaking sector}

One simple way to obtain supersymmetry breaking in local models is to introduce an appropriate kind of fractional brane in the local geometry. The resulting theory is no longer conformal, and in particular infrared dynamics generate a runaway superpotential that breaks supersymmetry. These kinds of branes (called DSB branes in the classification of [35]) are very generic, the simplest example appearing already in the complex cone over $d P_{1}$. For the moment we take this geometry, with the appropriate brane, to be our supersymmetry breaking sector (we will improve the problem of having a runaway potential in following sections). The relevant data for this sector is shown in figure 9 .

Let us review why this theory has a runaway potential $[35,36]$. We denote the fields going from $\mathrm{U}(M)$ to $\mathrm{U}(2 M)$ as $X_{12}, Y_{12}, Z_{12}$, the fields going from $\mathrm{U}(3 M)$ to $\mathrm{U}(M) X_{31}, Y_{31}$ and the field going from $\mathrm{U}(2 M)$ to $\mathrm{U}(3 M) X_{23}$. We can read off the superpotential for the theory from the dimer model in figure 9c:

$$
W=X_{23} X_{31} Y_{12}-X_{23} Y_{31} X_{12} .
$$

Note in particular that $Z_{12}$ is decoupled, and parameterizes a flat direction. Since this is only a toy model, we will ignore this issue in the following.

As we remarked in footnote 3 , generically the $\mathrm{U}(1)$ factors get masses by mixing with background RR fields. In the particular case we are dealing with all U(1) factors are anomalous (except for the overall $\mathrm{U}(1)$, which decouples), and thus necessarily get a mass. So we are in fact dealing with a $\mathrm{SU}(3 M) \times \mathrm{SU}(2 M) \times \mathrm{SU}(M)$ gauge theory.

Let us assume that the node of rank $3 M$ confines first. In this case the theory develops an ADS superpotential, given by [35-38]

$$
W=M_{21} Y_{12}-M_{21}^{\prime} X_{12}+M\left(\frac{\Lambda_{3}^{7 M}}{\operatorname{det} \mathcal{M}}\right)^{\frac{1}{M}},
$$

where we have introduced the mesons $M_{21}=X_{23} X_{31}, M_{21}^{\prime}=X_{23} Y_{31}$, and the mesonic $2 M \times 2 M$ matrix $\mathcal{M}=\left(M_{21} ; M_{21}^{\prime}\right)$. We can easily see the runaway direction from here: the F-term equations for $Y_{12}$ and $X_{12}$ want to set $M_{21}=M_{21}^{\prime}=0$. But now the F-term equation for the mesons pushes $Y_{12}$ and $X_{12}$ to infinity, due to the inverse power of the meson matrix appearing in the ADS term. 

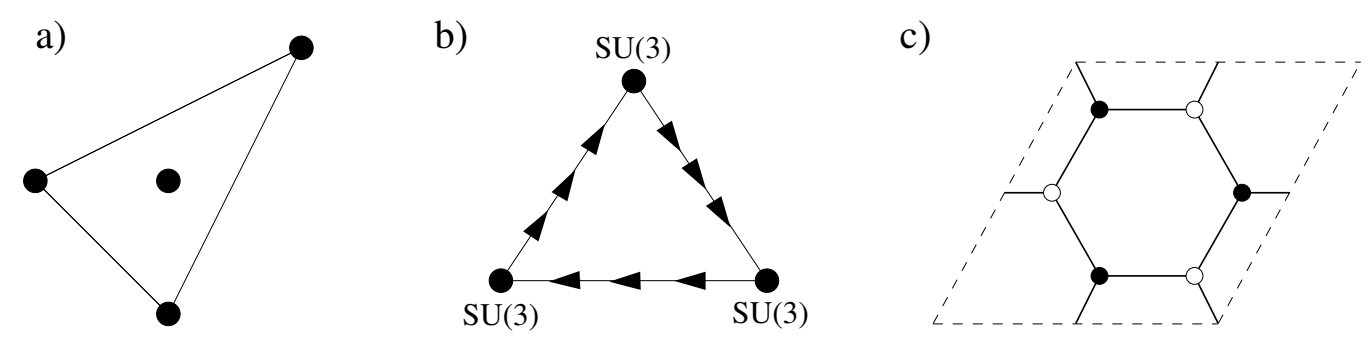

Figure 10. a) Toric diagram for the complex cone over $d P_{0}$. b) The quiver gauge theory for $3 \mathrm{D} 3$ branes at the singular point of $\mathbb{C}^{3} / \mathbb{Z}_{3}$. c) Dimer model, all faces have rank 3 .

It is possible to add a metastable vacuum at the origin of the runaway direction in moduli space by adding vector-like massive matter to our theory [39], see also [40] for generalizations to a large class of toric singularities. A simple way of achieving this is by introducing non-compact D7 branes that come close to the singularity. Open strings between the D3 branes at the singularity and the flavor D7 branes give rise to massive bifundamentals.

In our setup, we have another natural candidate for the massive flavors - the open strings between the supersymmetry breaking sector and the visible sector. This configuration is an interesting variation of the direct mediation [41, 42] family of models. (See also $[43,44]$ for early studies of direct mediation of metastable supersymmetry breaking.)

\subsection{The visible/dark matter sector}

For the visible and dark matter sectors we choose to put our branes at the singular point of the $\mathbb{C}^{3} / \mathbb{Z}_{3}$ orbifold, also known as the complex cone over $d P_{0}$. This gives the theory described in figure 10. These kinds of models with gauge group $\mathrm{SU}(3)^{3}$ and three families of bifundamentals are usually referred to as trinification models (see for example [45]). The idea of taking the dark matter sector to be a "mirror world", namely a copy of the standard model sector, also has a long history in phenomenology and it is a well studied possibility [29].

\subsection{Putting the different sectors together}

As we mentioned in the introduction to this section, part of the work of combining the three sectors was already done in [5], where one $d P_{1}$ and one $d P_{0}$ were joined together into a $X^{3,1}$ singularity, studied in $[22,46]$, see figure 11 . We are left to consider the last step of joining the $X^{3,1}$ singularity with the complex cone over $d P_{0}$. From the latter, we then get a new dark matter sector, given by a copy of the MSSM.

Following the discussion in section 3 , this is done by joining technique, placing the complex cone of $d P_{0}$ at a finite distance from the $X^{3,1}$ singularity. Let us consider the $X^{3,1}$ subsector. The corresponding dimer model is given in figure $11 \mathrm{~b}$. We draw the zig-zag paths of this model in figure 11c. In order to join the singularities, we need to remove the path corresponding to the internal leg (shown with a dashed line in figure 11c), and superpose the external legs coming from the $\mathbb{C}^{3} / \mathbb{Z}_{3}$ part of the geometry that we are attaching. As shown in figure $12 \mathrm{a}$, the external legs have slopes $(-1,-1)$ and $(-1,2)$. In figure $12 \mathrm{~b}$ we have superposed a couple of zig-zag paths with those winding numbers to the amputated 


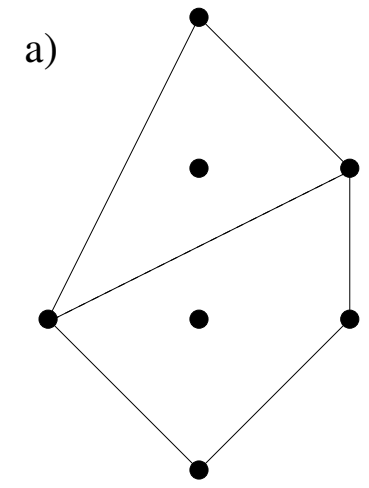

b)

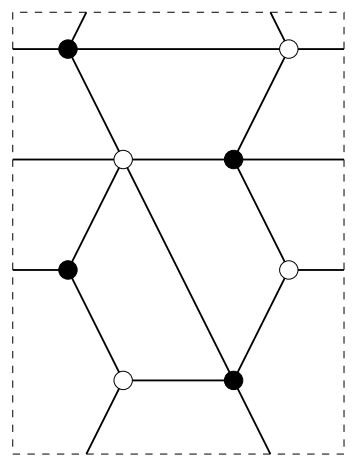

c)

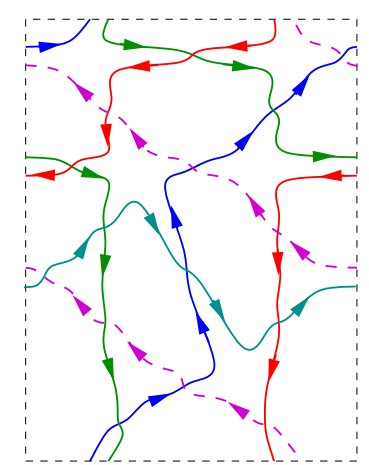

Figure 11. a) Toric geometry for the $X^{3,1}$ singularity, obtained by joining the $d P_{0}$ and $d P_{1}$ singularities along the dashed line. b) Dimer model. c) Harlequin diagram obtained from the dimer model. We have indicated with a dashed magenta line the zig-zag path along which we join $X^{3,1}$ to the $\mathbb{C}^{3} / \mathbb{Z}_{3}$ geometry.

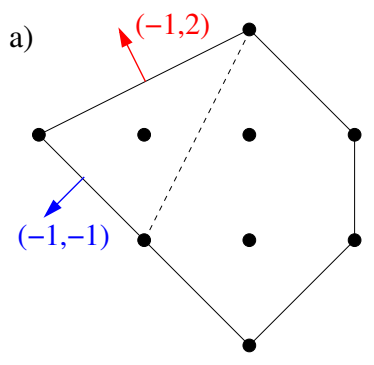

b)

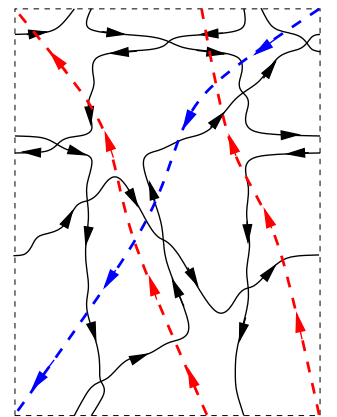

c)

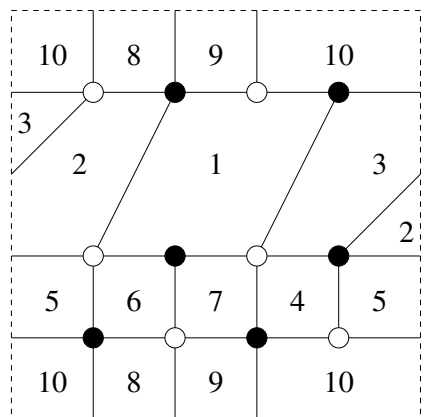

Figure 12. a) Toric geometry for the three sector model, obtained by joining the $X^{3,1}$ geometry to $d P_{0}$ along the dashed line. We have shown the external legs of the $d P_{0}$ sector that we are attaching. b) Harlequin diagram for the joined theory, obtained by adding the external zig-zag paths of $\mathbb{C}^{3} / \mathbb{Z}_{3}$ (dashed, in color) to the external zig-zag paths in harlequin diagram of figure 11c (in black). c) Reconstructed dimer model. We have numbered the faces for later reference.

harlequin diagram obtained from figure 11c. Finally, in figure 12c we have reconstructed the final dimer model from the set of zig-zag paths, as described in section 2.4.

We have included fractional branes in order to obtain a supersymmetry breaking sector, so in order to completely specify the model we also need to specify the ranks of the different faces in the dimer model. This is simple to determine once we have the explicit description of the resolution in terms of dimer models (see section 3.2). Each face of the mother dimer model maps to a specific face of the daughter dimer models. The rank of the face in the mother theory is simply the sum of the ranks of the corresponding faces in the daughter theory. That is, we have the following expression for the rank of the face $i$ in the mother dimer model:

$$
\operatorname{rank}\left(F_{i}\right)=\sum_{\text {daughterd }} \operatorname{rank}\left(F_{d}\left(F_{i}\right)\right)
$$

where $F_{d}\left(F_{i}\right)$ denotes the face in the daughter dimer model corresponding to $F_{i}$ in the mother. 


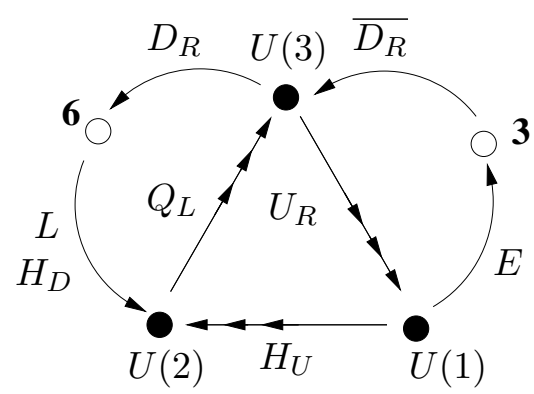

Figure 13. A better toy model for the MSSM, from [47]. The filled dark dots denote gauge groups, while the white dots denote global symmetry groups, coming from non-compact D7 branes.

The procedure is systematic, so let us just quote the result that we get. Numbering of the faces as in figure $12 \mathrm{c}$, the rank vector is given by:

$$
\vec{Q}=6 \cdot(1,1,1,1,1,1,1,1,1,1)+(M, 2 M, M, M, 0,3 M, 3 M, 2 M, 3 M, 2 M) .
$$

That is, our theory has gauge group $\mathrm{SU}(6+M) \times \mathrm{SU}(6+2 M) \times \mathrm{SU}(6+M) \times \ldots$. The first term comes from the regular branes at the $d P_{0}$ singularities, corresponding to the standard model and the dark matter sector (they contribute 3 D3 branes each), and the second term comes from the fractional brane at the $d P_{1}$ singularity. It is a simple exercise to check that there are no tadpoles with this assignment of ranks.

\section{Some interesting modifications of the construction}

In the previous section we have discussed the construction of a particular dark matter model. Our construction is highly modular, and this section will illustrate this point by discussing some interesting modifications of the structure given above. We have chosen examples that require introducing some extra ingredients into our construction.

\subsection{Flavor D7 branes and an improved standard model}

We can improve the visible sector using the model in [47], ${ }^{10}$ which is given by a fractional brane assignment in the $\mathbb{C}^{3} / \mathbb{Z}_{3}$ orbifold, with some non-compact D7 branes with baryonic charge. The quiver for this theory is shown in figure 13. It includes the usual three family MSSM (without right handed neutrinos), and some extra vector-like matter. Here hypercharge does not correspond to the $\mathrm{U}(1)$ node (which is anomalous), but rather to a non-anomalous combination of the abelian factors of the gauge symmetry on the nodes. In particular, it is given by:

$$
Y=\frac{1}{3} Q_{3}+\frac{1}{2} Q_{2}+Q_{1}
$$

where $Q_{N}$ represents the abelian part of the $\mathrm{U}(N)$ node.

Let us review for completeness how to introduce flavor in our theories using noncompact D7 branes passing through the singularity. In the following we will discuss massless

\footnotetext{
${ }^{10} \mathrm{~A}$ number of semi-realistic models involving branes at toric singularities and non-compact D7 branes have been recently described in [48].
} 


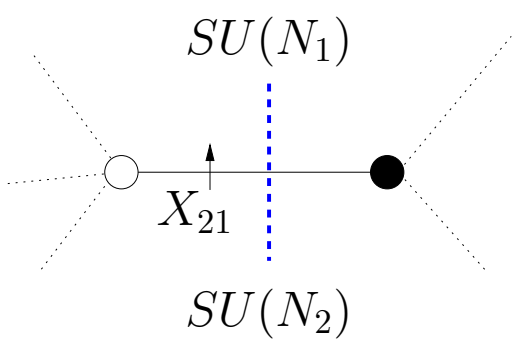

Figure 14. Local representation of a D7 brane, shown as a dashed blue line. $\mathrm{SU}\left(N_{1}\right)$ and $\mathrm{SU}\left(N_{2}\right)$ denote a couple of faces in the dimer model, $X_{21}$ is an edge connecting them, and the black and white nodes denote superpotential terms. The dotted lines represent extra edges in the dimer that do not enter our discussion.

flavors, but it is also possible to discuss massive flavors by introducing D7 branes that do not touch the singularity [49]. This is very convenient for building models of metastable supersymmetry breaking, see for example [39, 40].

D7 branes and dimer models. Let us start by briefly reviewing how to represent non-compact D7 branes in the language of dimer models [39].

Non-compact D7 branes wrap a non-compact divisor of the toric geometry. In terms of the web diagram, the basic class of non-compact divisors are associated with external legs. The basic idea is that we can think of the basic divisor $z_{i}=0$, where $z_{i}$ is a field in the gauge linear sigma model (GLSM), as determined by the couple of external legs of the web diagram bounding it. ${ }^{11}$

In the mirror of the toric variety, this D7 brane naturally projects to a curve wrapping a one cycle in a certain Riemann surface. We will explore this Riemann surface extensively in section 6, but let us postpone that discussion for now. The basic point is that this Riemann surface naturally encodes the dimer [27], and from the mirror description of the non-compact D7 brane we can read its description in dimer model terms. The end result is simple to state: D7 branes wrapped on basic divisors can be introduced as decoration in the dimer model given by a line going from a face to a neighboring face, crossing one bifundamental edge. ${ }^{12}$ We have shown this in figure 14 .

The D7 brane introduces a couple of chiral multiplets $X_{17}, X_{72}$ into the theory, charged in the bifundamental of the flavor D7-brane group and $\mathrm{SU}\left(N_{(1,2)}\right)$. There is also a new superpotential term given by:

$$
W_{D 7}=X_{72} X_{21} X_{17}
$$

where we are omitting the overall trace, as usual.

The improved MSSM given above is easily described in this language. We refer the reader to [5] for details of the final dimer model, and a two-sector GMSB model involving one such subsector.

\footnotetext{
${ }^{11}$ In general, apart from the geometric divisor class of a D7 brane, we also need to specify the value of possible Wilson lines on it. The dimer model also captures this information in a natural way.

${ }^{12}$ There is a simple generalization for D7 branes wrapping general divisors. They get mapped to general open paths in the dimer model [50, 51].
} 
a)

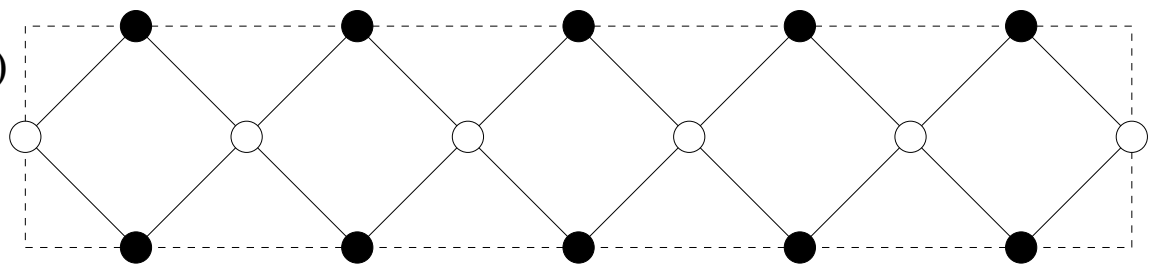

b)

c)

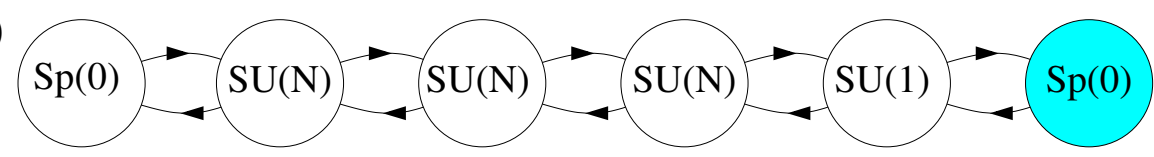

Figure 15. a) Dimer model for the $\mathbb{Z}_{5}$ orbifold of the conifold. b) Line orientifold [28] of the orbifold, giving the model used in [53]. We have denoted the orientifold plane by the vertical blue dashed line. We have also indicated the ranks of the faces. c) Quiver representation of the theory. The rightmost (colored) $\mathrm{Sp}(0)$ node gives rise to a mass term through a stringy instanton effect.

D7 branes and partial resolution. Upon partial resolution of the singularity the D7branes contribute additional matter. This gives one more massive messenger rule in addition to the four rules given in section 2.5:

5. For each D7-brane passing through an edge of type 1 there is a massive scalar multiplet in the fundamental representation of the $\mathrm{U}\left(N_{2}\right)$ gauge factor corresponding to the resulting recombined face. Similarly for edges of type 2 . When there are $N_{7} \mathrm{D} 7$ branes across such an edge the massive multiplet transforms as $\left(N_{D 3}, \overline{N_{7}}\right)$.

\subsection{Orientifolds and improved supersymmetry breaking}

The supersymmetry breaking sector described in the previous section has a couple of important shortcomings. First of all, it has a decoupled flat direction $Z$, which generically gets a mass after supersymmetry breaking (starting with two loops, since it is decoupled at the level of the superpotential), and might conceivably destabilize the metastable vacuum. Another important issue is that there is a runaway direction in the potential. While the metastable vacuum can be made long-lived, it is preferable to have a theory with a bona fide stable vacuum, in addition to the metastable one. In fact, various such configurations exist in the context of branes at singularities, see [52-55] for some simple examples that fit naturally in our toric framework. In this section we will show how we can replace the supersymmetry breaking sector in section 4 with the one proposed in [53]. Other cases can be shown to work similarly. 


\begin{tabular}{l|cccccc} 
& $a$ & $b$ & $c$ & $d$ & $e$ & $f$ \\
\hline $\mathbb{C}^{*}$ & 1 & 1 & -1 & -1 & 0 & 0 \\
$\mathbb{C}^{*}$ & 0 & 1 & 0 & 4 & -5 & 0 \\
$\mathbb{C}^{*}$ & 4 & 0 & 1 & 0 & 0 & -5
\end{tabular}

Table 1. GLSM description of the $\mathbb{Z}_{5}$ orbifold of the conifold.

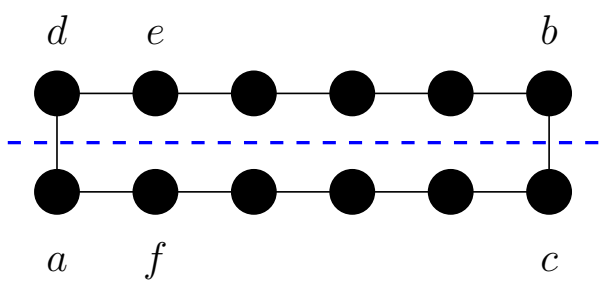

Figure 16. Toric diagram for the $\mathbb{Z}_{5}$ orbifold of the conifold. The dashed blue line denotes the action of the orientifold action on the geometry. We have also indicated which non-compact divisors of the toric geometry are associated with the fields in the GLSM.

Let us start by recalling the proposal of [53]. Consider a $\mathbb{Z}_{n}$ orbifold of the conifold, with $n \geq 5$. We take $n=5$, for simplicity. The relevant dimer model is shown in figure 15a. We also introduce an orientifold plane in the configuration, as shown in figure 15b. In [53] it was shown that such a configuration gives rise to a metastable long-lived ISS vacuum [43], in addition to the usual supersymmetric vacuum.

We choose the charges of the orientifold planes and the ranks of the faces such that we have an $\mathrm{Sp}(0) \times \mathrm{SU}(N) \times \mathrm{SU}(N) \times \mathrm{SU}(N) \times \mathrm{SU}(1) \times \mathrm{Sp}(0)$ gauge theory with vector-like matter. The different factors in the gauge group require some explanation. The SU(1) gauge group denotes a face where there is a single fractional D3 brane. The $\mathrm{Sp}(0)$ factor comes from a face mapped to itself under the orientifold action. The sign of the orientifold is chosen in such a way that it gives rise to $\operatorname{Sp}(M)$ factors in the gauge theory. In this example we put no fractional branes on the face, so we end up formally with a $\operatorname{Sp}(0)$ factor. As it is well understood by now from a variety of different viewpoints (see for example [53, 56-61], or [62] for a review), $\mathrm{Sp}(0)$ gauge groups give rise to non-perturbative dynamics in string theory due to $O(1)$ D-brane instantons wrapping the $\mathrm{Sp}(0)$ node. In our case, the effect of the instanton is to give a small mass to the bifundamental fields between the $\mathrm{SU}(1)$ node and its neighboring $\mathrm{SU}(N)$ node.

Let us see how we can construct a three sector model with this geometry as the supersymmetry breaking sector, replacing the complex cone over $d P_{1}$. Our first task is to understand how the orientifold acts on the geometry. We can determine this as follows. Consider the GLSM description of the $\mathbb{Z}_{5}$ orbifold of the conifold described in table 1 . The corresponding toric diagram is shown in figure 16. Note that the external nodes of the toric diagram, which correspond to non-compact divisors of the toric geometry, are associated with fields in the GLSM. As an example, the upper right hand node of the toric diagram in figure 16 corresponds to the $b=0$ divisor. 


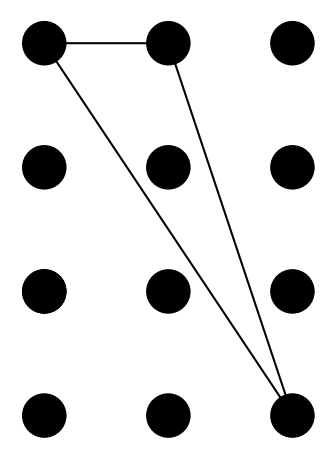

Figure 17. Toric diagram of $\mathbb{C}^{3} / \mathbb{Z}_{3}$ after applying (5.6).

The gauge invariants describing the geometry are:

$$
\begin{aligned}
x & =a^{5} d^{5} e^{4} f^{4} \\
y & =b^{5} c^{5} e f \\
z & =a c f \\
w & =b d e
\end{aligned}
$$

with the constraint $x y=z^{5} w^{5}$. As discussed in [28], the line orientifold in figure 15 acts as:

$$
z \leftrightarrow w, \quad x \rightarrow x, \quad y \rightarrow y
$$

We can easily read the action on the GLSM fields $a, b, c, d$ from here, it is given by:

$$
a \leftrightarrow d, \quad b \leftrightarrow c, \quad e \leftrightarrow f
$$

Looking to the toric diagram in figure 16, this implies that the orientifold acts as a reflection of the toric diagram along the dashed line. We will present further evidence for this action, from the point of view of mirror symmetry, in section 6.3.

Let us now describe how to join the supersymmetry breaking sector to the visible and dark matter sectors. As in previous sections, we model the latter by two copies of $\mathbb{C}^{3} / \mathbb{Z}_{3}$. Looking to figure $10 \mathrm{a}$, it is clear that we need to perform a $\mathrm{SL}(2, \mathbb{Z})$ transformation in order to be able to join the subsectors. It is most convenient to transform the $\mathbb{C}^{3} / \mathbb{Z}_{3}$ geometry using the following $\mathrm{SL}(2, \mathbb{Z})$ action:

$$
T=\left(\begin{array}{cc}
0 & -1 \\
1 & 1
\end{array}\right)
$$

This transformation brings the toric diagram in figure 10a into the form shown in figure 17.

Now we can easily attach the top of figure 17 to the bottom of 16 . Due to the action of the orientifold, we also need to attach a mirror copy on the top. The result of doing this (including also another copy of $\mathbb{C}^{3} / \mathbb{Z}_{3}$ for the dark matter sector) is shown in figure 18 .

First, notice that we cannot just join the three sectors and obtain a well defined toric geometry, since the resulting toric diagram would not be convex. This is easily remedied 


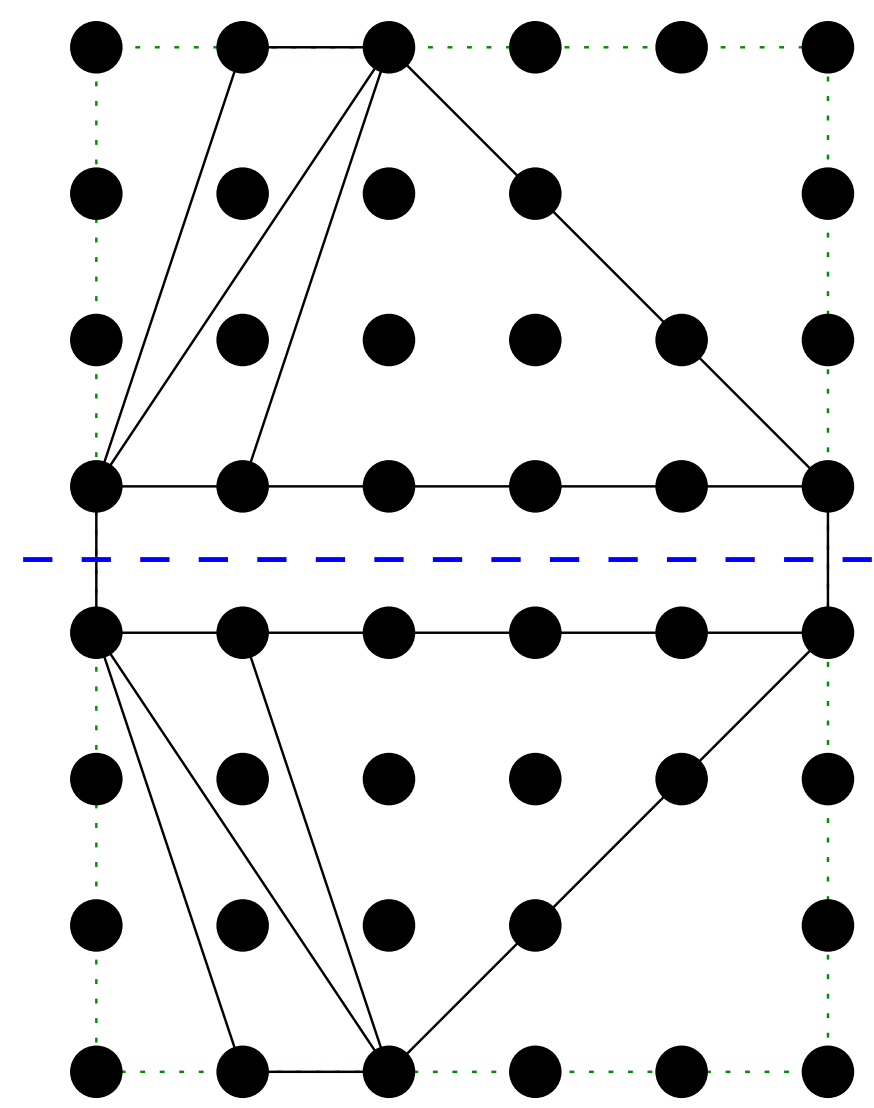

Figure 18. Three sector moose with an improved supersymmetry breaking subsector. We have denoted the orientifold point by the blue dashed horizontal line. The green dotted rectangle around the figure denotes the $\mathbb{Z}_{5} \times \mathbb{Z}_{7}$ orbifold of the conifold which can be used to obtain the gauge theory of our model, as explained in the text.

by adding the piece in the lower right of figure 18 (and its image on the top right), which corresponds to a $\mathbb{C}^{3} /\left(\mathbb{Z}_{3} \times \mathbb{Z}_{4}\right)$ orbifold of flat space. When assigning the ranks of the faces of the daughter dimer models we can choose to put no branes in the daughter diagram corresponding to this extra orbifold, so this part of the geometry does not contribute any massless degrees of freedom. Nevertheless it modifies the details of the resulting theory, in particular the couplings to the massive sector fields.

Even if the total geometry is complicated, it is actually straightforward to work out the dimer model description for the joined singularity without having to go through the joining algorithm. The basic observation is that the singularity that we get can be easily embedded into a $\mathbb{Z}_{5} \times \mathbb{Z}_{7}$ orbifold of the conifold, as shown by the dotted line in figure 18 . Taking orbifolds of dimer models is very simple, and reduces to increasing the size of the unit cell [63]. Going from the orbifold of the conifold to our desired geometry involves removing a $\mathbb{C}^{2} / \mathbb{Z}_{3} \times \mathbb{C}$ on the top left (and its image), and a $\mathbb{C}^{3} /\left(\mathbb{Z}_{3} \times \mathbb{Z}_{3}\right)$ piece on the top right (and its image). This is done in the dimer using the resolution algorithm described in section 2.5, so we skip the detailed discussion. 


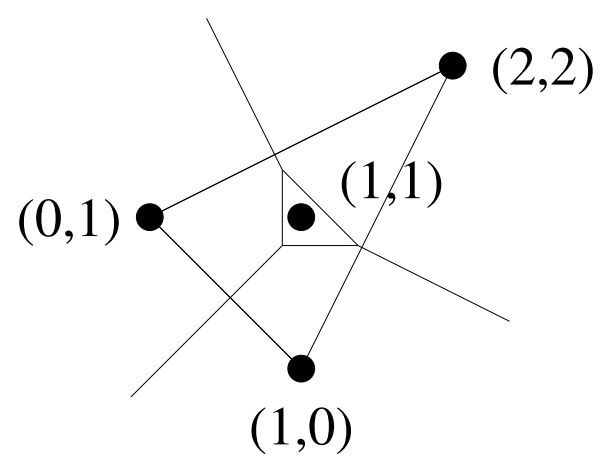

Figure 19. Toric diagram and web diagram for the $\mathbb{C}^{3} / \mathbb{Z}_{3}$ orbifold, also known as the complex cone over $d P_{0}$. The labels denote the positions in the square lattice of the nodes in the toric diagram.

\section{Mirror description of the small resolution}

So far we have focused on the type IIB picture, reviewing the set of rules that describe the small resolution in terms of a Higgsing of the field theory living on the branes located at the singularity.

Our type IIB system has a well known mirror description [64, 65]. In fact, as was shown in [27], the dimer model construction admits a very natural interpretation in the mirror picture. The effect of the small resolution on the field theory reviewed above can also be motivated from the mirror [4]. In this section we describe the mirror type IIA intersecting brane system, and in particular discuss in some detail how to obtain the complex structure deformation that is mirror to the small resolution we want to perform in type IIB.

The dimer model is most naturally seen as a hybrid description of the system in the sense that it encodes the relevant information for both sides of the mirror. Any statement that we make in terms of the dimer model can be easily read in the mirror type IIA language, and allows us to explicitly implement any of our modular constructions also in the class of intersecting brane models mirror to branes at toric singularities. ${ }^{13}$

\subsection{The mirror manifold}

Let us review in this section the construction of the mirror manifold, referring the reader to the original references $[27,64,65]$ for more details.

Take any toric Calabi-Yau variety, described by a polytope in the two-dimensional square lattice. For illustrative purposes we choose the complex cone over $d P_{0}$, shown in figure 19. It is useful to distinguish between external and internal points in the toric diagram. In the case of $d P_{0}$ we have the external points $(1,0),(0,1),(2,2)$, and a single internal point at $(1,1) \cdot{ }^{14}$

The type IIA mirror manifold is given by a double fibration over the complex plane $\mathbb{C}$. Let us denote the coordinate in the complex plane as $W$. The equations defining the

\footnotetext{
${ }^{13}$ See for example [66] for an example of some interesting model building possibilities using type IIA geometries similar to the ones that we construct.

${ }^{14}$ Toric diagrams without internal points require extra care [27, 28]. Except for one of our examples in section 6.2 , we work with toric singularities with internal points.
} 
fibration are:

$$
\begin{aligned}
u v & =W \\
P(z, w) & =W
\end{aligned}
$$

where $u, v \in \mathbb{C}$ and $z, w \in \mathbb{C}^{*}$. Here, $P(z, w)$, the Newton polynomial, is given by the equation

$$
P(z, w)=\sum_{(p, q) \in D} c_{(p, q)} z^{p} w^{q}
$$

where the sum is over the positions of the points in the lattice belonging to the toric diagram (including possible internal points), and $c_{(p, q)}$ are arbitrary complex numbers parameterizing the complex structure moduli space of the mirror manifold. For $d P_{0}$ we get the following Newton polynomial:

$$
P(z, w)=c_{(1,0)} z+c_{(0,1)} w+c_{(1,1)} z w+c_{(2,2)} z^{2} w^{2} .
$$

Notice that there is an ambiguity in the definition of the polynomial, since we have to choose a particular origin of the lattice, and a $\mathrm{SL}(2, \mathbb{Z})$ frame for the embedding. These choices give rise to isomorphic geometries, since they both arise from the underlying $\operatorname{SL}(3, \mathbb{Z})$ ambiguity of the toric description of the singularity. Acting with this $\mathrm{SL}(3, \mathbb{Z})$ changes the coordinates which we use to describe the GLSM formulation of the toric singularity, but it does not change the toric space itself.

Equation (6.1) describes a $\mathbb{C}^{*}$ that degenerates over $W=0$ into two intersecting complex planes, while the most interesting part of the geometry comes from (6.2). This equation describes a Riemann surface, $\Sigma$, of genus equal to the number of internal points in the toric diagram, fibered over the complex plane $W . \Sigma$ is actually non-compact, having one puncture for each external leg of the web diagram. Note that this surface admits a nice description as a thickening of the web diagram, an observation that we will use extensively in section 6.2. For an illustration of the thickening in the case of $\mathbb{C}^{3} / \mathbb{Z}_{3}$, see figure 20 .

The Riemann surface $\Sigma$ degenerates at $n$ critical points, $W=W_{*}$, where $n$ is the number of triangles in the toric diagram. For example, for $\mathbb{C}^{3} / \mathbb{Z}_{3}$ we have that the toric diagram is made of 3 elementary triangles, so we expect (6.4) to degenerate at 3 points. It is a simple matter to check that $\partial_{z} P(z, w)=\partial_{w} P(z, w)=P(z, w)-W=0$ does indeed have three different solutions for the three unknowns $(z, w, W)$. Notice that all the information about where in the base $\Sigma$ degenerates is encoded in the $c_{(p, q)}$ coefficients, so giving these is sufficient to specify the geometry completely. Because of this fact, in the discussion given below we will focus only on $\Sigma$ itself, with the understanding that this information is enough to define a mirror Calabi-Yau manifold via the construction given above.

Before proceeding with mapping Kähler deformations to complex structure deformations in the mirror manifold, for completeness let us say a few words about the mirror description of the (fractional) D3 branes. As discussed above, there are $n$ points $W_{*}$ on the base where some one-cycle in $\Sigma$ degenerates. We also have that the $S^{1}$ circle in the $\mathbb{C}^{*}$ fiber degenerates over $W=0$. If we connect $W=0$ to any of the $n$ points $W_{*}$ by a segment in the $W$ plane, and consider the total space of the fibration given by the two 


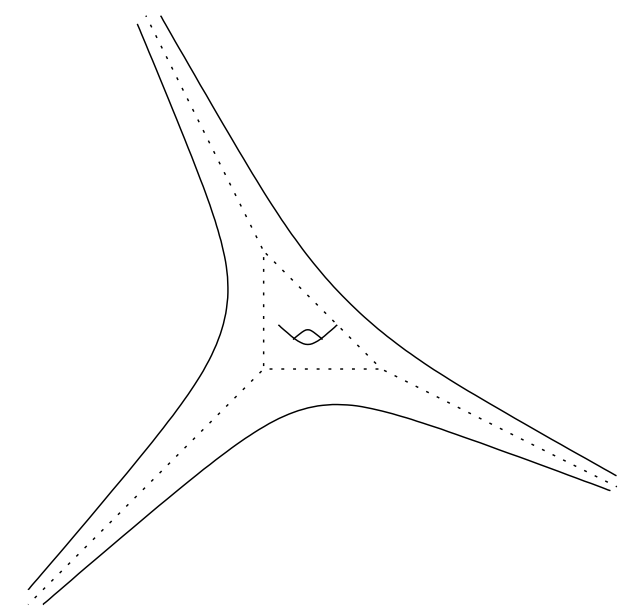

Figure 20. Thickening of the $\mathbb{C}^{3} / \mathbb{Z}_{3}$ web diagram, giving the mirror Riemann genus 1 surface $\Sigma$. We have also superposed, using dotted lines, the skeleton web diagram.

$S^{1}$ circles degenerating at the boundary of the segment, we obtain a $S^{3}$. Since we have $n$ critical points $W_{*}$, we have $n$ such three-spheres. The mirror of the $n$ different fractional branes in the original geometry ${ }^{15}$ are the different D6 branes wrapping these $S^{3}$ cycles. The dimer model then arises from the intersection of the $S^{3}$ cycles over $W=0$, and in particular can be understood purely from intersections of one-cycles over $\Sigma$. We refer the reader to [27] for further details on how this construction works, and how to understand many of the features of the dimer model from it.

\subsection{The amoeba map and small resolutions}

Up to now we have described the mirror at a general point in complex structure moduli space, without yet specifying the values of the $c_{(p, q)}$ coefficients in the Newton polynomial $P(z, w)$. Recall that we are interested in Kähler deformations that partially smooth out the singularity, meaning that we blow up some internal two- (or four-) cycles, leaving separated daughter singularities. In general, mapping Kähler deformations to complex structure deformations of the mirror is a complicated problem. Fortunately, as we have seen there is a deep relation between $\Sigma$ and the web diagram of our singularity, and this allows us to see which complex deformations do the job of splitting the Riemann surface into two daughter Riemann surfaces with the right properties.

A tool that we find particularly useful is the amoeba map [67-71], defined as the image in $\mathbb{R}^{2}$ of the points of $\Sigma$ (defined as the locus $P(z, w)=0$ ) under:

$$
(z, w) \rightarrow(\log |z|, \log |w|)
$$

We illustrate the result of applying this map to the complex cone over $d P_{0}$ in figure 21 .

An important property of the amoeba is that its tentacles align with the external legs of the web diagram (see figure 21). It is useful for us to work through some examples

\footnotetext{
${ }^{15}$ Remember that $n$ is the number of triangles in the toric diagram, which coincides with the number of nodes of the quiver.
} 


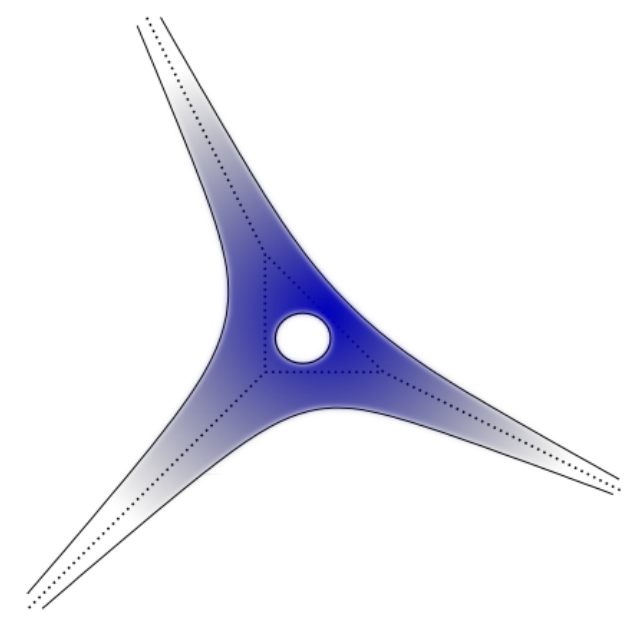

Figure 21. Amoeba for the Riemann surface mirror to the $\mathbb{C}^{3} / \mathbb{Z}_{3}$ orbifold. We have indicated the web diagram skeleton by the dotted line.

explicitly, so let us show this for the $d P_{0}$ singularity. (For a general proof, see [27].) Let us take the following expression for the Newton polynomial defining $\Sigma$ :

$$
P(z, w)=z+w+d z w+z^{2} w^{2}=0,
$$

where we have used the freedom to redefine the coordinates in order to make three coefficients equal to 1 . The Kähler deformation parameter of $\mathbb{C}^{3} / \mathbb{Z}_{3}$ is encoded in the value for $d$. Let us focus on the lower left leg of the web diagram, see figure 19. It is perpendicular to the edge going from $(1,0)$ to $(0,1)$ in the toric diagram. It is easy to see that there is such a tentacle of the amoeba: it is given by the family of solutions $z=-w \rightarrow 0$ (where we discarded higher order terms in (6.6)). Under the amoeba map, $(z,-z)$ with $z \rightarrow 0$ maps to $(t, t)$ with $t \rightarrow-\infty$, reproducing the proper spike.

The other two tentacles work in a similar way. Let us focus on the tentacle going northwest. The corresponding leg in the web diagram is associated with the edge going from $(0,1)$ to $(2,2)$. In terms of the Newton polynomial, these are the $w$ and $z^{2} w^{2}$ terms. Let us thus write the Newton polynomial equation in the following way:

$$
P(z, w)=w\left(1+z^{2} w\right)+z+d z w=0 .
$$

Consider the branch of the solution with $w \rightarrow \infty$. In order to keep the product in the first term finite, we need $z^{2}=-1 / w \rightarrow 0$. Note that the last term $d z w$, although divergent, is subleading and can be ignored when computing the asymptotic behavior of the amoeba. We end up with the following curve of solutions: $\left(z,-1 / z^{2}\right)$ with $z \rightarrow 0$, which maps to $(t,-2 t)$ with $t \rightarrow-\infty$, matching the result from the web diagram. Finally, in order to obtain the south-east tentacle, write the curve as:

$$
P(z, w)=z\left(1+z w^{2}\right)+w+d z w=0 .
$$

There is a branch of solutions with $z \rightarrow \infty$ and $z w^{2}=-1$, in other words $\left(-1 / w^{2}, w\right)$ with $w \rightarrow 0$, mapping to $(2 t,-t)$ with $t \rightarrow \infty$. 
The previous discussion can be seen as a way of "undoing mirror symmetry", in the sense that the skeleton of the amoeba is given by the web diagram, and represents faithfully the original toric geometry. It is a natural question whether the same projection can be used to map complex structure moduli deformations to Kähler moduli deformations. As we will see in examples below, this is actually possible. In particular, we will argue that it is possible to identify which complex structure deformations are dual to which Kähler deformations splitting the original singularity into two smaller singularities.

Before explaining in some selected examples how this works, let us mention a couple of limitations of the method we will present momentarily. First of all, we are computing properties of the asymptotics of the amoeba, so we do not see any Kähler deformation that leaves these invariant. Consider the $\mathbb{C}^{3} / \mathbb{Z}_{3}$ geometry we have been using to illustrate our discussion. As we saw, the $d$ parameter is subleading, and does not influence the asymptotics. This is also easy to see from the web diagram: we can blow up the collapsed 4-cycle keeping the external legs fixed. Luckily, the kinds of the small resolutions that we deal with in this paper are not of this kind: when we partially blow up the singularity the external legs get displaced. This is in fact the basic idea of our method: we can compute this displacement easily and reliably looking to the asymptotics. Whichever complex deformation displaces the tentacles of the amoeba in the right way gets identified with the mirror of the Kähler blow-up mode.

Secondly, when computing the behavior of the external tentacles the coefficients of internal points are often subleading, and hard to determine looking only to asymptotics. In complicated examples we just assume that we know their correct value so they reproduce the right mirror for the singular daughter geometries. This is not a big problem for us, since we are mostly interested in giving an explicit description of how to deform the mirror so we end up with various decoupled (at the massless level) sectors. Knowing which moduli we have to send to 0 or $\infty$ is enough for understanding this.

With these limitations in mind, let us discuss how we can understand the geometry of the mirror better in a simple way in a few interesting examples.

The conifold. We start by considering our favorite example, the conifold. Its Newton polynomial equation is:

$$
P(z, w)=1+z+w z+a w=0
$$

where we have defined the variables in such a way that the last first coefficients are equal to 1 . The complexified resolution parameter of the conifold is then identified with the complex structure modulus $a$. Let us see how we can see this using the amoeba projection. The west spike of the amoeba is associated with the $1+a w$ term. We can satisfy (6.9) by setting $z \rightarrow 0, w=-1 / a$. Under the amoeba map, this maps to the line $(t,-\log |a|)$ with $t \rightarrow-\infty$. It is easy to work out the locations of the other tentacles in a similar way, the 

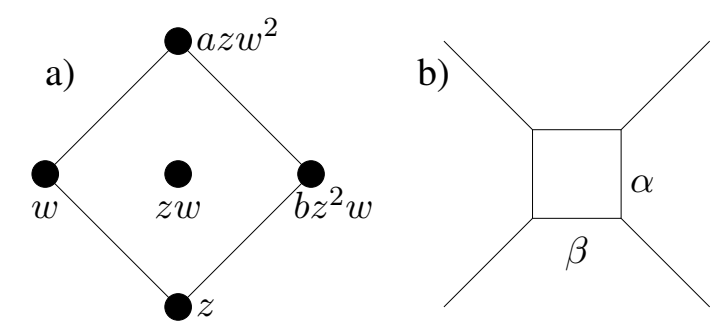

Figure 22. a) Toric diagram for the complex cone over $\mathbb{F}_{0}$. We have indicated the corresponding terms in the Newton polynomial (6.15). b) Web diagram. The $\alpha$ and $\beta$ parameters correspond to the size of the two independent blow-up modes of $\mathbb{F}_{0}$.

result is:

$$
\begin{aligned}
\text { South: } & (0, t), \quad t \rightarrow-\infty \\
\text { West: } & (t,-\log |a|), \quad t \rightarrow-\infty \\
\text { North: } & (\log |a|, t), \quad t \rightarrow \infty \\
\text { East: } & (t, 0), \quad t \rightarrow \infty
\end{aligned}
$$

Note that once we send $\log |a| \rightarrow-\infty$ this exactly reproduces the web diagram description (given in figure 6a) of the resolution of the conifold into two copies of flat space. This tells us that the right limit of (6.9) to reproduce the splitting is $a \rightarrow 0$, and more generally that we can identify the resolution parameter $v$ of the conifold with $-\log |a|$.

This is in fact also natural from the point of view of the toric diagram. Sending $a \rightarrow 0$ takes (6.9) into

$$
P(z, w)=1+z+w z=0
$$

which reproduces the equation obtained from the toric diagram of flat space. This result is general, at least for the examples that we will be dealing with, and can be stated very simply as follows: in order to obtain the mirror of the resolved geometry, send to 0 the coefficients of the Newton polynomial located purely on one side of the resolution.

The complex cone over $\mathbb{F}_{\mathbf{0}}$. Let us check this rule in a couple of further examples. The first example we want to discuss is the complex cone over $\mathbb{F}_{0}=\mathbb{P}^{1} \times \mathbb{P}^{1}$. The toric and web diagrams are shown in figure 22.

The Newton polynomial equation defining the geometry can be taken to be:

$$
P(z, w)=z+w+z w+a z w^{2}+b z^{2} w
$$

We can determine the slope and displacement of the external legs easily using the same methods as above. For example, in order to compute the displacement of the south-east tentacle, we need to center on the $z+b z^{2} w$ term in the Newton polynomial. Rewriting (6.15) as:

$$
P(z, w)=z(1+b z w)+w+z w+a z w^{2}
$$

we obtain a tentacle of the amoeba by sending $z \rightarrow \infty$ while keeping $w=-1 / b z$. That is, we obtain the line given by $(t,-t-\log |b|)$ with $t \rightarrow \infty$. We proceed similarly for the other 
a)

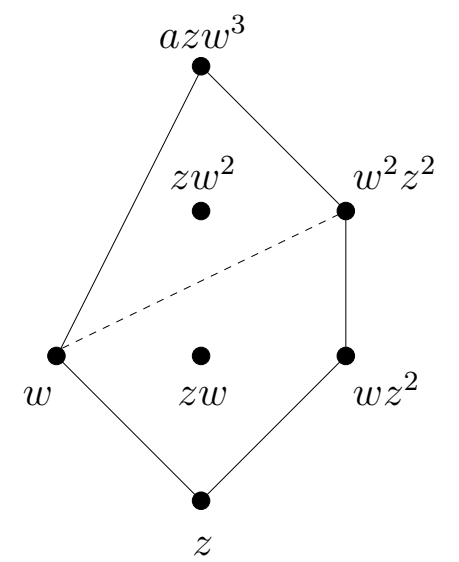

b)

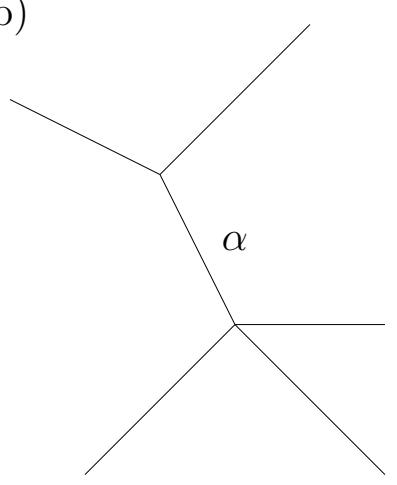

Figure 23. a) Toric diagram for the MSSM and susy breaking sectors in section 4 . We have indicated the desired resolution mode by the dashed lines. We have also indicated which terms in (6.21) correspond to which vertex. b) Corresponding web diagram. Note that we have not resolved the internal four-cycles in order to make better contact with the text. We have denoted the size of the resolved two-cycle by $\alpha$.

external legs, obtaining:

$$
\begin{aligned}
\text { South-West: } & (t, t), \quad t \rightarrow \infty \\
\text { South-East: } & (t-\log |b|,-t) \quad t \rightarrow \infty \\
\text { North-East: } & (t-\log |b|, t-\log |a|) \quad t \rightarrow \infty \\
\text { North-West: } & (-t, t-\log |a|) \quad t \rightarrow \infty
\end{aligned}
$$

It is easy to see that this is precisely the structure of external legs in the resolved web diagram shown in figure $22 \mathrm{~b}$, once we identify $\alpha=-\log |a|$ and $\beta=-\log |b|$. This also matches beautifully with the rule described above: if we wanted to resolve the geometry into a couple of $\mathbb{C}^{2} / \mathbb{Z}_{2} \times \mathbb{C}$ singularities we tune the Kähler moduli to be $\alpha=0, \beta \rightarrow \infty$. This is precisely $a=1, b=0$, which removes the leftmost node in the toric diagram in figure 22a from the Newton polynomial (6.15).

The $\boldsymbol{X}^{(\mathbf{3}, 1)}$ geometry. Let us discuss another example, given by the mirror of the $X^{(3,1)}$ geometry we used in section 4 for modelling the MSSM and susy breaking sectors in our three sector dark matter model. We reproduce in figure 23 the relevant toric and web diagrams.

We have a Newton polynomial given by:

$$
P(z, w)=w+a z w^{3}+w^{2} z^{2}+w z^{2}+z=0
$$

where we have omitted the terms corresponding to the internal points, since they do not modify the asymptotics. As described above, we expect that setting $a \rightarrow 0$ splits the Riemann surface into the two desired daughter Riemann surfaces. We obtain the following 
asymptotics for the tentacles of the amoeba:

$$
\begin{aligned}
\text { North-West: } & (-2 t-\log |a|, t), \quad t \rightarrow \infty \\
\text { North-East: } & (t+\log |a|, t), \quad t \rightarrow \infty \\
\text { East: } & (0, t), \quad t \rightarrow \infty \\
\text { South-East: } & (t,-t), \quad t \rightarrow \infty \\
\text { South-West: } & (-t,-t), \quad t \rightarrow \infty
\end{aligned}
$$

It is easy to check that these lines reproduce the structure of the resolved web diagram for $X^{(3,1)}$ shown in figure $23 \mathrm{~b}$, once we set $\alpha=-\log |a| / 3$. In particular, the NW and NE external legs intersect over $(-\alpha, 2 \alpha)$ in the plane where the amoeba is defined. The rest of the legs intersect over $(0,0)$.

Let us discuss an important point that we have ignored until now, and which will become useful in a moment. Notice that in doing the blow up both daughter sub-sectors enter on an equal footing, while our construction seems to depend strongly on which subsector contains the point that we want to "remove" by sending $a \rightarrow 0$. However, there is a way of rewriting things in such a way that the same resolution can be described by sending the coefficients of the points on the other side of the resolution to zero. Let us describe how this works in our $X^{(3,1)}$ example. The key observation is that the position of the origin of the amoeba plane is conventional, so in particular we should be able to scale our $z, w$ variables in order to put the NW-NE intersection at the origin. The relevant scaling is given by $\tilde{z}=z / \tilde{a}, \tilde{w}=w \tilde{a}^{2}$ with $\tilde{a}^{3}=a$. In terms of these variables the Newton polynomial equation becomes:

$$
P(\tilde{z}, \tilde{w})=\frac{1}{\tilde{a}^{2}}\left(\tilde{z} \tilde{w}^{3}+\tilde{w}+\tilde{w}^{2} \tilde{z}^{2}\right)+\tilde{a} \tilde{z}+\tilde{w} \tilde{z}^{2}=0 .
$$

Multiplying the whole equation by $\tilde{a}^{2}$ we get:

$$
\tilde{P}(\tilde{z}, \tilde{w})=\tilde{z} \tilde{w}^{3}+\tilde{w}+\tilde{w}^{2} \tilde{z}^{2}+\tilde{a}^{3} \tilde{z}+\tilde{a}^{2} w \tilde{z}^{2}=0 .
$$

Notice how the resulting polynomial now agrees with the general prescription when applied to the other side of the resolution: setting $\tilde{a} \rightarrow 0$ removes the terms in the Newton polynomial corresponding to that side of the resolution, and indeed resolves the singularity as desired. In this case the powers of $\tilde{a}$ are non-trivial, and are determined by imposing that the external legs corresponding to the $d P_{1}$ part of the geometry intersect at a single point.

The dark matter model. Just for completeness, let us include here the result for the mirror of our three-sector dark matter model from section 4 . The relevant toric diagram is shown in figure 24. The Newton polynomial equation (omitting internal points, as usual) is given by:

$$
P(z, w)=b w^{2}+z^{2} w^{3}+z w+w^{2} z^{3}+a^{3} z^{2}+a^{2} w z^{3}=0 .
$$

The discussion proceeds just as in the examples given above, and we obtain the factorized three-sector geometry by sending $a \rightarrow 0, b \rightarrow 0$. 


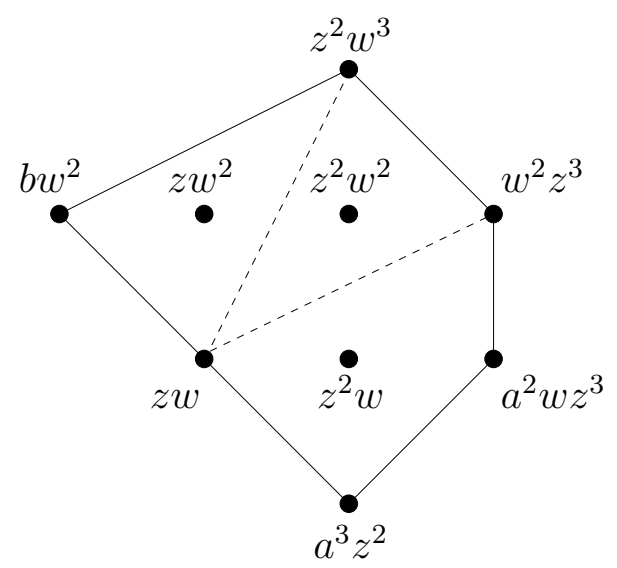

Figure 24. Toric diagram for the dark matter model in section 4 . We have indicated the desired resolutions by dashed lines, and we have associated monomials in (6.24) to their corresponding edges.

As we have seen in the previous examples, looking to the displacement of the tentacles via the amoeba map gives a simple way of determining how to deform the Newton polynomial in order to split the system into decoupled sub-sectors. Our construction gives a simple and very intuitive procedure for determining how to deform the Riemann surface.

\subsection{Orientifolds and algae}

In section 5.2 we discussed the geometric action of the orientifold in the toric diagram, or equivalently in the web diagram, and argued from the defining GLSM that the proper geometric action is a reflection along a horizontal line. Let us shortly describe how the mirror picture supports this identification.

We described in the previous section how the amoeba map gives a way to map the behavior of the Riemann surface to the mirror description in terms of the web diagram. There is a complementary projection of the Riemann surface called the alga projection, defined by:

$$
(z, w)=\left(|z| e^{i \alpha},|w| e^{i \beta}\right) \rightarrow(\alpha, \beta)
$$

This projection defines a region on a two torus that turns out to be a thickening of the dimer model [27]. Let us remark that the dimer model that we obtain is naturally "aligned" with the web diagram, in the sense that if a leg of the web diagram goes in a $(p, q)$ direction in the amoeba plane, then the corresponding zig-zag path wraps a $(p, q)$ cycle in the dimer model $T^{2}$.

As described in [28], the above observation can be used to understand the behavior of the mirror surface under the orientifold action. The basic idea is that the phases of $z, w$ must transform as the coordinates of the dimer $T^{2}$. Since we know the action of the orientifold on the dimer we can infer its action on the phases of $z, w$. Combined with the fact that the orientifold action on the mirror must be anti-holomorphic, we can determine how $z, w$ transform under the orientifold. 
Consider the configuration studied in section 5.2. The line orientifold reflects the dimer horizontally, while leaving it invariant vertically. An anti-holomorphic involution that does this is [28]:

$$
\begin{gathered}
z \rightarrow \bar{z} \\
w \rightarrow \frac{1}{\bar{w}}
\end{gathered}
$$

Notice that the phase of $z$ changes sign, while the phase of $w$ does not, according to the fact that the reflection of the dimer model is along the horizontal direction.

It is easy to see the action that (6.26) induces on the amoeba. It is given by:

$$
(\log |z|, \log |w|)=(s, t) \rightarrow(s,-t)
$$

The horizontal reflection has turned into a vertical one. Reading the action on the web diagram from the action on the amoeba, this exactly reproduces the geometric action found in section 5.2.

\section{Conclusions and open questions}

We have described a general procedure for joining arbitrary theories coming from branes at toric singularities into a consistent local embedding which couples them via massive mediators. We have applied this technique for building a three sector model of dark matter consisting of the standard model, a dark matter sector, and a supersymmetry breaking sector, all interacting via massive mediators of a tunable scale. We have also constructed some alternative models in order to illustrate the flexibility of our constructions. In particular, note that some of our examples can be naturally adapted (by forgetting about the dark matter sector) to become GMSB scenarios with a nicely behaved susy breaking sector. Finally, we have described the mirror picture of our construction, giving us a procedure for joining a large class of intersecting brane configurations (specifically, those mirror to branes at toric singularities).

There are various interesting directions in which our results can be extended. The most obvious one is to generalize our construction to models of branes at non-toric singularities. In particular, the case of branes at singular non-toric del Pezzos can be analyzed using exceptional collections [72, 73]. It would be interesting to develop techniques to analyze these cases in a way similar to the one we have developed in this paper. ${ }^{16}$ Being able to handle higher del Pezzo singularities would allow for some very interesting model building possibilities [33, 75].

Another related line of research concerns the relation to recent model building work based on local F-theory constructions (see [76-80] for some of the original papers). Most of the subsectors in this paper are based on blown down del Pezzo surfaces. The same kind of geometry, but blown up and having a different choice of wrapped brane, can be used to

\footnotetext{
${ }^{16}$ The first four complex cones over del Pezzo are actually toric, and can be understood both from the point of view of exceptional collections and dimer models. Furthermore, for these cases the map between the two approaches is well understood by now [74].
} 
construct semi-realistic models of the MSSM GUTs in F-theory. It would be interesting to construct "hybrid" models, having the visible sector coming from a D7 wrapping a large blown-up del Pezzo, while having branes at singularities nearby providing for susy breaking and interacting dark matter. In fact some of these hybrid models have already been studied in the literature [3].

The models that we have constructed are based on small resolutions, and are thus controlled by Kähler moduli. In our local constructions these are flat directions. We have assumed that we can tune the Kähler moduli as we wish, but in any realistic setting one must construct a detailed moduli stabilization scenario. This depends on global features of our model, and is thus beyond the reach of the tools discussed in this paper. Related to this last point, we expect the global embedding of our construction to introduce interesting constraints in the local physics. It would thus be useful to have a better understanding of possible global completions of our models.

There is an alternative to our construction which improves on this moduli stabilization problem, at the cost of making the models less calculable. The idea is to trigger geometric transitions by putting confining D-branes on the singularity. Once the branes confine, we end up with daughter singularities separated by three cycles stabilized by the dual flux. Similar techniques to the ones presented in this paper apply to this scenario $[4,5,35]$. The problem with this setup is that one losses calculability of the mediator sector, which is now determined by the spectrum of states in the confining theory. This is a well defined question, but the answer is very hard to determine with available tools.

As we discussed in section 6 , our analysis carries over easily to the context of intersecting branes in type IIA. Under mirror symmetry, the resolutions that we have constructed in type IIB map to complex structure deformations of the type IIA background. In type IIA it is possible to stabilize complex structure moduli by the introduction of $\mathrm{H}_{3}$ and geometric flux [81-84], so the problem of moduli stabilization may in principle be ameliorated. Unfortunately, studying in detail the backreaction of the flux on the type IIA geometry is an involved problem, and thus it is hard to construct explicit models with all moduli stabilized.

Finally, in this paper we have focused mainly on developing tools, and understanding the construction better via some toy models. It would be interesting to construct more realistic models of interacting dark matter and/or gauge mediated supersymmetry breaking using these tools, and analyze their phenomenology in more detail.

\section{Acknowledgments}

We are happy to acknowledge interesting and fruitful discussions with Mirjam Cvetič, Angel Uranga and Tomer Volansky. We would also like to thank Antonio Amariti, Luciano Girardello and Alberto Mariotti for informing us of their research on a related topic [17]. V.B. thanks the Yukawa Institute for Theoretical Physics for hospitality while this work was being completed. P.B. thanks the organizers of the workshop "New Perspectives in String Theory" at the Galileo Galilei Institute for Theoretical Physics, Florence, where early stages of this work was carried out. I.G.-E. thanks the CERN Theory Division for 
hospitality, and Nao Hasegawa for kind support and constant encouragement. The work of P.B. is supported by the NSF CAREER grant PHY-0645686 and by the University of New Hampshire through its Faculty Scholars Award Program. I.G.-E.'s work is supported by the High Energy Physics Research Grant DE-FG05-95ER40893-A020.

Open Access. This article is distributed under the terms of the Creative Commons Attribution Noncommercial License which permits any noncommercial use, distribution, and reproduction in any medium, provided the original author(s) and source are credited.

\section{References}

[1] G.F. Giudice and R. Rattazzi, Theories with gauge-mediated supersymmetry breaking, Phys. Rept. 322 (1999) 419 [hep-ph/9801271] [SPIRES].

[2] N. Arkani-Hamed, D.P. Finkbeiner, T.R. Slatyer and N. Weiner, A theory of dark matter, Phys. Rev. D 79 (2009) 015014 [arXiv:0810.0713] [SPIRES].

[3] D.-E. Diaconescu, B. Florea, S. Kachru and P. Svrček, Gauge - mediated supersymmetry breaking in string compactifications, JHEP 02 (2006) 020 [hep-th/0512170] [SPIRES].

[4] I. Garcia-Etxebarria, F. Saad and A.M. Uranga, Quiver gauge theories at resolved and deformed singularities using dimers, JHEP 06 (2006) 055 [hep-th/0603108] [SPIRES].

[5] I. Garcia-Etxebarria, F. Saad and A.M. Uranga, Local models of gauge mediated supersymmetry breaking in string theory, JHEP 08 (2006) 069 [hep-th/0605166] [SPIRES].

[6] H. Verlinde, L.-T. Wang, M. Wijnholt and I. Yavin, A higher form (of) mediation, JHEP 02 (2008) 082 [arXiv: 0711.3214] [SPIRES].

[7] T.W. Grimm and A. Klemm, U(1) mediation of flux supersymmetry breaking, JHEP 10 (2008) 077 [arXiv: 0805.3361] [SPIRES].

[8] R. Rattazzi, C.A. Scrucca and A. Strumia, Brane to brane gravity mediation of supersymmetry breaking, Nucl. Phys. B 674 (2003) 171 [hep-th/0305184] [SPIRES].

[9] K. Choi, A. Falkowski, H.P. Nilles and M. Olechowski, Soft supersymmetry breaking in KKLT flux compactification, Nucl. Phys. B 718 (2005) 113 [hep-th/0503216] [SPIRES].

[10] S. Kachru, L. McAllister and R. Sundrum, Sequestering in string theory, JHEP 10 (2007) 013 [hep-th/0703105] [SPIRES].

[11] M. Buican and S. Franco, SUSY breaking mediation by D-brane instantons, JHEP 12 (2008) 030 [arXiv:0806.1964] [SPIRES].

[12] F. Benini et al., Holographic gauge mediation, JHEP 12 (2009) 031 [arXiv:0903.0619] [SPIRES].

[13] V.S. Kaplunovsky and J. Louis, Model independent analysis of soft terms in effective supergravity and in string theory, Phys. Lett. B 306 (1993) 269 [hep-th/9303040] [SPIRES].

[14] A. Brignole, L.E. Ibáñez and C. Muñoz, Towards a theory of soft terms for the supersymmetric Standard Model, Nucl. Phys. B 422 (1994) 125 [Erratum ibid. B 436 (1995) 747] [hep-ph/9308271] [SPIRES].

[15] N. Seiberg, Electric-magnetic duality in supersymmetric nonAbelian gauge theories, Nucl. Phys. B 435 (1995) 129 [hep-th/9411149] [SPIRES]. 
[16] B. Feng, A. Hanany and Y.-H. He, D-brane gauge theories from toric singularities and toric duality, Nucl. Phys. B 595 (2001) 165 [hep-th/0003085] [SPIRES].

[17] A. Amariti, L. Girardello and A. Mariotti, Pseudomoduli dark matter and quiver gauge theories, arXiv:0910.3615 [SPIRES].

[18] K.D. Kennaway, Brane tilings, Int. J. Mod. Phys. A 22 (2007) 2977 [arXiv:0706.1660] [SPIRES].

[19] M. Yamazaki, Brane tilings and their applications, Fortsch. Phys. 56 (2008) 555 [arXiv: 0803.4474] [SPIRES].

[20] N.C. Leung and C. Vafa, Branes and toric geometry, Adv. Theor. Math. Phys. 2 (1998) 91 [hep-th/9711013] [SPIRES].

[21] I.R. Klebanov and E. Witten, Superconformal field theory on threebranes at a Calabi-Yau singularity, Nucl. Phys. B 536 (1998) 199 [hep-th/9807080] [SPIRES].

[22] S. Franco, A. Hanany, K.D. Kennaway, D. Vegh and B. Wecht, Brane dimers and quiver gauge theories, JHEP 01 (2006) 096 [hep-th/0504110] [SPIRES].

[23] L.E. Ibáñez, R. Rabadán and A.M. Uranga, Anomalous U(1)'s in type-I and type IIB $D=4$, $N=1$ string vacua, Nucl. Phys. B 542 (1999) 112 [hep-th/9808139] [SPIRES].

[24] D.R. Morrison and M.R. Plesser, Non-spherical horizons. I, Adv. Theor. Math. Phys. 3 (1999) 1 [hep-th/9810201] [SPIRES].

[25] R. Kenyon and J.-M. Schlenker, Rhombic embeddings of planar graphs with faces of degree 4, Trans. Am. Math. Soc. 357 (2005) 3443.

[26] A. Hanany and D. Vegh, Quivers, tilings, branes and rhombi, JHEP 10 (2007) 029 [hep-th/0511063] [SPIRES].

[27] B. Feng, Y.-H. He, K.D. Kennaway and C. Vafa, Dimer models from mirror symmetry and quivering amoebae, Adv. Theor. Math. Phys. 12 (2008) 3 [hep-th/0511287] [SPIRES].

[28] S. Franco et al., Dimers and orientifolds, JHEP 09 (2007) 075 [arXiv:0707.0298] [SPIRES].

[29] R. Foot and R.R. Volkas, Neutrino physics and the mirror world: How exact parity symmetry explains the solar neutrino deficit, the atmospheric neutrino anomaly and the LSND experiment, Phys. Rev. D 52 (1995) 6595 [hep-ph/9505359] [SPIRES].

[30] N. Arkani-Hamed and N. Weiner, LHC signals for a superUnified theory of dark matter, JHEP 12 (2008) 104 [arXiv:0810.0714] [SPIRES].

[31] R. Blumenhagen, M. Cvetič and T. Weigand, Spacetime instanton corrections in $4 D$ string vacua - the seesaw mechanism for D-brane models, Nucl. Phys. B 771 (2007) 113 [hep-th/0609191] [SPIRES].

[32] L.E. Ibáñez and A.M. Uranga, Neutrino Majorana masses from string theory instanton effects, JHEP 03 (2007) 052 [hep-th/0609213] [SPIRES].

[33] M. Buican, D. Malyshev, D.R. Morrison, H. Verlinde and M. Wijnholt, D-branes at singularities, compactification and hypercharge, JHEP 01 (2007) 107 [hep-th/0610007] [SPIRES].

[34] L.E. Ibáñez and A.M. Uranga, Instanton induced open string superpotentials and branes at singularities, JHEP 02 (2008) 103 [arXiv:0711.1316] [SPIRES].

[35] S. Franco, A. Hanany, F. Saad and A.M. Uranga, Fractional branes and dynamical supersymmetry breaking, JHEP 01 (2006) 011 [hep-th/0505040] [SPIRES]. 
[36] K.A. Intriligator and N. Seiberg, The runaway quiver, JHEP 02 (2006) 031 [hep-th/0512347] [SPIRES].

[37] D. Berenstein, C.P. Herzog, P. Ouyang and S. Pinansky, Supersymmetry breaking from a Calabi-Yau singularity, JHEP 09 (2005) 084 [hep-th/0505029] [SPIRES].

[38] M. Bertolini, F. Bigazzi and A.L. Cotrone, Supersymmetry breaking at the end of a cascade of Seiberg dualities, Phys. Rev. D 72 (2005) 061902 [hep-th/0505055] [SPIRES].

[39] S. Franco and A.M. Uranga, Dynamical SUSY breaking at meta-stable minima from D-branes at obstructed geometries, JHEP 06 (2006) 031 [hep-th/0604136] [SPIRES].

[40] I. Garcia-Etxebarria, F. Saad and A.M. Uranga, Supersymmetry breaking metastable vacua in runaway quiver gauge theories, JHEP 05 (2007) 047 [arXiv: 0704.0166] [SPIRES].

[41] I. Affleck, M. Dine and N. Seiberg, Dynamical supersymmetry breaking in four-dimensions and its phenomenological implications, Nucl. Phys. B 256 (1985) 557 [SPIRES].

[42] M.A. Luty and J. Terning, Improved single sector supersymmetry breaking, Phys. Rev. D 62 (2000) 075006 [hep-ph/9812290] [SPIRES].

[43] K.A. Intriligator, N. Seiberg and D. Shih, Dynamical SUSY breaking in meta-stable vacua, JHEP 04 (2006) 021 [hep-th/0602239] [SPIRES].

[44] R. Kitano, H. Ooguri and Y. Ookouchi, Direct mediation of meta-stable supersymmetry breaking, Phys. Rev. D 75 (2007) 045022 [hep-ph/0612139] [SPIRES].

[45] S. Willenbrock, Triplicated trinification, Phys. Lett. B 561 (2003) 130 [hep-ph/0302168] [SPIRES].

[46] A. Hanany, P. Kazakopoulos and B. Wecht, A new infinite class of quiver gauge theories, JHEP 08 (2005) 054 [hep-th/0503177] [SPIRES].

[47] G. Aldazabal, L.E. Ibáñez, F. Quevedo and A.M. Uranga, D-branes at singularities: A bottom-up approach to the string embedding of the standard model, JHEP 08 (2000) 002 [hep-th/0005067] [SPIRES].

[48] J.P. Conlon, A. Maharana and F. Quevedo, Towards realistic string vacua, JHEP 05 (2009) 109 [arXiv:0810.5660] [SPIRES].

[49] A. Karch and E. Katz, Adding flavor to AdS/CFT, JHEP 06 (2002) 043 [hep-th/0205236] [SPIRES].

[50] Y. Imamura, K. Kimura and M. Yamazaki, Anomalies and O-plane charges in orientifolded brane tilings, JHEP 03 (2008) 058 [arXiv:0801.3528] [SPIRES].

[51] D. Forcella, I. Garcia-Etxebarria and A. Uranga, E3-brane instantons and baryonic operators for D3-branes on toric singularities, JHEP 03 (2009) 041 [arXiv:0806.2291] [SPIRES].

[52] R. Argurio, M. Bertolini, S. Franco and S. Kachru, Gauge/gravity duality and meta-stable dynamical supersymmetry breaking, JHEP 01 (2007) 083 [hep-th/0610212] [SPIRES].

[53] R. Argurio, M. Bertolini, S. Franco and S. Kachru, Metastable vacua and D-branes at the conifold, JHEP 06 (2007) 017 [hep-th/0703236] [SPIRES].

[54] M. Buican, D. Malyshev and H. Verlinde, On the geometry of metastable supersymmetry breaking, JHEP 06 (2008) 108 [arXiv:0710.5519] [SPIRES].

[55] A. Amariti, D. Forcella, L. Girardello and A. Mariotti, Metastable vacua and geometric deformations, JHEP 12 (2008) 079 [arXiv:0803.0514] [SPIRES]. 
[56] K.A. Intriligator, P. Kraus, A.V. Ryzhov, M. Shigemori and C. Vafa, On low rank classical groups in string theory, gauge theory and matrix models, Nucl. Phys. B 682 (2004) 45 [hep-th/0311181] [SPIRES].

[57] L.E. Ibáñez, A.N. Schellekens and A.M. Uranga, Instanton induced neutrino majorana masses in CFT orientifolds with MSSM-like spectra, JHEP 06 (2007) 011 [arXiv: 0704.1079] [SPIRES].

[58] R. Argurio, M. Bertolini, G. Ferretti, A. Lerda and C. Petersson, Stringy instantons at orbifold singularities, JHEP 06 (2007) 067 [arXiv:0704.0262] [SPIRES].

[59] M. Bianchi, F. Fucito and J.F. Morales, D-brane instantons on the $T^{6} / Z_{3}$ orientifold, JHEP 07 (2007) 038 [arXiv: 0704.0784] [SPIRES].

[60] M. Aganagic, C. Beem and S. Kachru, Geometric transitions and dynamical SUSY breaking, Nucl. Phys. B 796 (2008) 1 [arXiv:0709.4277] [SPIRES].

[61] I. Garcia-Etxebarria, D-brane instantons and matrix models, JHEP 07 (2009) 017 [arXiv:0810.1482] [SPIRES].

[62] R. Blumenhagen, M. Cvetič, S. Kachru and T. Weigand, D-brane instantons in Type II orientifolds, Ann. Rev. Nucl. Part. Sci. 59 (2009) 269 [arXiv:0902.3251] [SPIRES].

[63] A. Hanany and K.D. Kennaway, Dimer models and toric diagrams, hep-th/0503149 [SPIRES].

[64] K. Hori and C. Vafa, Mirror symmetry, hep-th/0002222 [SPIRES].

[65] K. Hori, A. Iqbal and C. Vafa, D-branes and mirror symmetry, hep-th/0005247 [SPIRES].

[66] A.M. Uranga, Local models for intersecting brane worlds, JHEP 12 (2002) 058 [hep-th/0208014] [SPIRES].

[67] G. Mikhalkin, Real algebraic curves, the moment map and amoebas, Ann. Math. 2 (2000) 151.

[68] G. Mikhalkin, Amoebas of algebraic varieties, (2001).

[69] H. Rullgård, Topics in geometry, analysis and inverse problems, Ph.D. thesis, Stockholm University, Stockholm, Sweden (2003).

[70] R. Kenyon, A. Okounkov, and S. Sheffield, Dimers and amoebae, (2003).

[71] G. Mikhalkin, Amoebas of algebraic varieties and tropical geometry, (2004).

[72] F. Cachazo, B. Fiol, K.A. Intriligator, S. Katz and C. Vafa, A geometric unification of dualities, Nucl. Phys. B 628 (2002) 3 [hep-th/0110028] [SPIRES].

[73] M. Wijnholt, Large volume perspective on branes at singularities, Adv. Theor. Math. Phys. 7 (2004) 1117 [hep-th/0212021] [SPIRES].

[74] A. Hanany, C.P. Herzog and D. Vegh, Brane tilings and exceptional collections, JHEP 07 (2006) 001 [hep-th/0602041] [SPIRES].

[75] H. Verlinde and M. Wijnholt, Building the Standard Model on a D3-brane, JHEP 01 (2007) 106 [hep-th/0508089] [SPIRES].

[76] R. Donagi and M. Wijnholt, Model building with F-theory, arXiv:0802.2969 [SPIRES].

[77] C. Beasley, J.J. Heckman and C. Vafa, GUTs and exceptional branes in F-theory - I, JHEP 01 (2009) 058 [arXiv: 0802.3391] [SPIRES]. 
[78] H. Hayashi, R. Tatar, Y. Toda, T. Watari and M. Yamazaki, New aspects of heterotic-F theory duality, Nucl. Phys. B 806 (2009) 224 [arXiv:0805.1057] [SPIRES].

[79] C. Beasley, J.J. Heckman and C. Vafa, GUTs and exceptional branes in F-theory - II: experimental predictions, JHEP 01 (2009) 059 [arXiv:0806.0102] [SPIRES].

[80] R. Donagi and M. Wijnholt, Breaking GUT groups in F-theory, arXiv:0808.2223 [SPIRES].

[81] J.-P. Derendinger, C. Kounnas, P.M. Petropoulos and F. Zwirner, Superpotentials in IIA compactifications with general fluxes, Nucl. Phys. B 715 (2005) 211 [hep-th/0411276] [SPIRES].

[82] S. Kachru and A.-K. Kashani-Poor, Moduli potentials in type IIA compactifications with RR and NS flux, JHEP 03 (2005) 066 [hep-th/0411279] [SPIRES].

[83] G. Villadoro and F. Zwirner, $N=1$ effective potential from dual type- IIA D6/O6 orientifolds with general fluxes, JHEP 06 (2005) 047 [hep-th/0503169] [SPIRES].

[84] J.P. Derendinger, C. Kounnas, P.M. Petropoulos and F. Zwirner, Fluxes and gaugings: $N=1$ effective superpotentials, Fortsch. Phys. 53 (2005) 926 [hep-th/0503229] [SPIRES]. 